\title{
A responsiveness metric for the design and planning of resilient supply chains
}

\author{
João Pires Ribeiro' ${ }^{1}$ - Ana Paula F. D. Barbosa-Póvoa ${ }^{1}$
}

Accepted: 3 January 2022

(c) The Author(s), under exclusive licence to Springer Science+Business Media, LLC, part of Springer Nature 2022

\begin{abstract}
Supply Chain Management is in constant evolution, and Supply Chain Resilience (SCR) appears as a recent offspring result of changes in how companies do business. Research efforts on the topic have led to a focus on the basic concepts of SCR, leaving a relevant research gap on the modelling and quantification of the SCR behaviour. In fact, there is not yet a consensus on SCR metrics or on how to quantify SCR. Most SCR models fail to incorporate relevant characteristics of the supply chain's performance, as are the impacts perceived by downstream customers. This work addresses such gaps, and a new resilient SC metric is proposed, which is incorporated into a developed optimisation model, where economic and responsiveness objectives are maximised when designing and planning resilient SC considering all SC entities. The model is applied to a case study that shows that decisionmakers should avoid adopting universal strategies when managing their SC and instead should define the best plan for their SC operation. The impacts perceived by downstream customers are analysed. Moreover, it can be concluded that there is a correlation between the SC performance and the new SCR metric, easing the process of designing and planning the $\mathrm{SC}$ when resilience concerns are at stake.
\end{abstract}

Keywords Supply chain management - Supply chain resilience $\cdot$ Quantitative models . Metrics

\section{Introduction}

Supply Chain (SC) has emerged as the backbone of the global economy, with many economic activities heavily relying on such operations for its success. The dependency on these systems has led to the emergence of a distinct field of study, Supply Chain Management (SCM) (Oliver \& Webber et al. 1982). This activity implies the study of the main concerns inside SC operations and SCR, in particular, arises as a novel approach to manage the consequences of high-impact events on SC. History provides us with clear examples of how unforeseen circumstances can impact SC, and how different reactions can dictate profitability or, in the

João Pires Ribeiro

pires.ribeiro@tecnico.pt

1 CEG-IST, Instituto Superior Técnico, Universidade de Lisboa, Av. Rovisco Pais, 1, 1049-001 Lisbon, Portugal 
worst case, the dissolution of a business. This is the case of natural or anthropogenic disasters or even economic or political decisions.

In this work, we aim to address an adequate SCR metric to be used in a model that can support the design and planning of SC. Thus, meeting one research gap in this field (Ribeiro and Barbosa-Póvoa 2018b). Most published articles leave the SCR to qualitative interpretations (Das 2018), and few look into quantitative analysis (Sokolov et al. 2016; Cardoso et al. 2015). A quantitative approach is pursued in this paper by exploring financial aspects coupled with service level goals, modelled as a SCR metric that is used in the design and planning of resilient SC when uncertainty is present. To show the applicability of such approach, a mixed-integer linear programming model (MILP) is proposed, where different SC networks are studied, from only forward to closed-loop supply chains (CLSC), the latter encompassing circular economy principles. The results show the justifiability of such approaches towards the incorporation of resilience into supply chains, providing decision-makers with a broader insight into SCR as well as a means to improve SC operations.

The features that distinguish this paper from the existing literature include the quantitative study of SCR on a strategic-tactical level of decision, focusing on designing and planning a resilient global CLSC. A generic multi-product, multi-period and multistage MILP model is presented, accounting for uncertainty in demand and disruptive scenarios. This includes the development of a new metric, meeting in this way a relevant research gap identified by academics and practitioners - the need to find adequate measures to supply chain resilience. This metric combines the profit goal with the capacity of guaranteeing high service levels under disruptive scenarios. The application of this metric and the promising results are illustrated in Sect. 6.

The novel approach provides a new path for SCR, providing not only a new tool to be used on the design and planning of resilient supply chains but a quantitative model that explores prior conceptualizations of SCR, such are the four pillars of SCR presented by (Ribeiro and Barbosa-Póvoa 2018b) (Focus Event, Performance Level, Speed and Adaptive Framing). The developed tool allows for the study of different disruptive events, providing the usage of a new performance level that leads to the presentation of alternative adaptive framing solutions to each SC/disruptive scenario. Due to the nature of a strategic model, the speed on how the $\mathrm{SC}$ responds to disruptive scenarios is not considered in this work.

The outcomes of this paper, namely the new SCR metric, the mathematical model and the managerial insights, can be used by both academics and practitioners when looking at their operations. The features of SCR here explored, as is the case of meeting customer demand or economic return, have been traditionally presented individually. This work is one of the few attempts that integrate these aspects to provide a more holistic and usable tool for SCR.

The remainder of this paper is structured as follows: Sect. 2 presents the work being done in SCR modelling; Sect. 3 presents the problem description; Sect. 4 characterises the mathematical model; Sect. 4.2 presents the new SCR metric; Sect. 5 presents the disruptive scenarios; Sect. 6 presents and solves a case study; Sect. 7 performs discussion and conclusion of results; Sect. 8 presents managerial insights. Finally, Sect. 9 concludes the work and proposes future directions.

\subsection{Resilience in SC}

Natural disasters are one example of events that can severely impact SC Duhamel et al. (2016). The consequences of Hurricane Katrina were far more persistent than the natural 
phenomenon. The destruction it caused culminated in stoppages in the oil refining industry and on the production of products like fruit or lumber (Snyder et al. 2006; Vigdor 2008).

Areas prone to earthquakes are also an example of how severe the SC consequences of an unpredictable event can be. The auto industry, partially due to high investment in lean practices, is highly sensitive to changes in the steady-state operation, with disturbances being able to cause long-lasting adverse effects. This sensitivity was verified in 2011 when Japan's auto industry was severely affected by an earthquake. The resulting stoppages cost millions of dollars, and 11 months were required to restore production levels (Takahashi 2011).

No matter the event that leads to the disruption, some lessons can be learned on how to manage and cope better with this particular kind of events (Wachtendorf, Brown, and Holguin-Veras 2013; Wakasugi and Tanaka 2015). Even simpler weather events, such as high gusts, heavy rain, or extreme temperatures, can induce effects on the company's operation. Minimising the risk for the whole SC is thus of great importance (Brusset and Bertrand 2018).

On this, relationship amongst SC actors and how they affect each other has been studied in several fields. In SCR, one can study the impact of the type of contracts between suppliers and production in the response to disruptions (Li et al. 2017, 2020). Information sharing between the different players can suffice for the appropriate SC response to disturbances (Sabahi and Parast 2020; Tao, Lai, and Zhou 2020). Positive relationships can foster responsiveness and efficiency in the SC (Goffnett and Williams 2019); on the contrary, confrontations can have negative impacts. The strike at two General Motors plants led to a gridlock in the SC, resulting in losses of millions of dollars (Snyder et al. 2006), another example of how a single event can cause a ripple effect through the system.

One of the most referred examples of the importance of resilient capabilities and information sharing amongst the SC players is the example of the fire in 2000 at a Philips factory that impacted two big players, Nokia and Ericsson. This fire forced a stop in production, and despite being informed at the same time, the two firms had very different responsive actions and final results. Nokia was fast to acknowledge the disruption and to seek alternatives, Ericsson failed to do so and ended up having around 400 million \$ in losses (Dolgui, Ivanov, and Sokolov 2018). The lack of agility leads to the inability to remain competitive (Sharma et al. 2017), which is a lack of reaction after the occurrence of a stimulus (Bernardes and Hanna 2009).

The concept of SCR is therefore very important and can be applied to a broad spectrum of economic activities with examples in several sectors, including: chemical (Cardoso et al. 2015); automotive (Carvalho et al. 2012); agri-business (Stone and Rahimifard 2018; Kirwan, Maye, and Brunori 2017; Mwangi et al. 2021), since all sectors can be affected by disruptions which could be internal, external or a combination of both. Although, disruptions can occur in any location of a SC, most commonly the study is centred around upstream disruptions. Transportation disruptions are often seen as one of the most frequent hurdles in SC operations. Albertzeth et al. (2020) studies such kind of scenarios and presents a metric to help find strategies to improve SCR, also tackling the difficult process of monetising in this context.

One of the dangers of improperly addressing SCR is failing to acknowledge the ripple effect that different events can have in the SC (Dolgui, Ivanov, and Sokolov 2018; Ribeiro and Barbosa-Póvoa 2019). The ripple effect is associated with the occurrence of one seemingly harmless event which can cause a complete shutdown in a domino effect. Despite possibly starting under the radar, companies must be capable of stopping and mitigating its propagation and for that should have resilient SC.

Recently, the world has faced the perfect storm in terms of disruptions, the COVID-19 pandemic, that evidenced the need for resilient SC. The restrictions and health consequences of the coronavirus outbreak were global and spared almost no country. There is no economic 
sector that left unscathed from this period. While some companies had their operation left to a complete standstill, others had a massive increase in demand. Moreover, some were in a compromised situation with part of their operations restricted.

The academic response was fast and researchers provided short publications aimed at exploring how science could help. The interconnected global SC were forced to face new scenarios and deal with the consequences (Baldwin and Tomiura 2020). Hobbs (2020) focus on the Canadian food industry and how it was impacted, both for disruptions on operations but also by the panic buying that brought a considerable demand variation. Mathematical approaches provide useful insights and Paul and Chowdhury (2020) presents a model on the operational level where the results add validity to the relevance of SCR, since SC designed with Resilience in mind had a better behaviour under stress, at least reducing costs.

The shortages of essential medical goods, eg. Personal Protective Equipment (PPE), in western countries are an evidence of policies that failed to implement a strategic resilient SC in-place (Gereffi 2020). This wake-up is leading to an increased concern on the topic and on the study of Resilient SC. Until now, re-shoring was seen as an option that would yield lower profits, companies are now considering it to mitigate shortages of essential products (Chen and Hu 2017). Regardless, the works on SCR its modelling and quantification have not reached a consensus on the best approach for resilience metrics (Golan, Jernegan, and Linkov 2020). However, SCM with a strong resilience drive is an option for decision makers to empower their companies. For that to be possible, academics must present concise, simple, and reliable models and metrics to provide useful managerial insights for decision makers.

\subsection{Relationship with other fields of SC}

In recent years, the world has been witnessing a shift in the way business is performed, enabled by higher connectivity along with advances in technology, shaped by new intense societal challenges such as the case of sustainability. This new paradigm for doing business brought a spotlight to activities not used to be the focal point of SCM. It calls for high SC effectiveness and responsiveness, a continuous challenge for companies (Ralston and Blackhurst 2020; Mohammed et al. 2021).

The increased awareness of sustainable SC leads to changes in all levels of SCM, creating new challenges. One of those is how can one achieve a resilient and sustainable SC (Mari, Lee, and Memon 2014; Fahimnia and Jabbarzadeh 2016). The re-use of products is on the rise at a global scale, leading to more complex SC and with it reverse logistics/closed-loop SC (Savaskan, Bhattacharya, and Van Wassenhove 2004; Howard, Hopkinson, and Miemczyk 2018; Özçelik et al.Özçelik et al. 2020).

Regardless of the recent increased interest in SCR, there is still space for further development and to create awareness and knowledge on how companies can overcome disruptive events (Ribeiro and Barbosa-Póvoa 2018b). Dealing with disruptive events must be done resorting to a holistic approach, where SCR has a lot to gain by taking advantage of the knowledge and experience provided by other SC fields that handle uncertainty and risk. Examples of such benefit are the application of the concept of reliability (Ha, Jun, and Ok 2018) or Supply Chain Risk Management (SCRM). One can even consider SCR as an offspring of SCRM (Ponomarov and Holcomb 2009; König and Spinler 2016; Shekarian and Mellat Parast 2020). Risk management consists of managing proactive practices taking into account vulnerability. Those can be prospective strategies to enhance SCR (Rajesh and Ravi 2015). Inspired by the biological immune system Srinivasan and Tew (2017) present a SCRM framework that can be adapted to SCR. 
However, to correctly face SCR adaptations are needed since the traditional methods of dealing with risk rely vastly on the identification and consequent quantification of probabilities and impacts of a particular event. An antagonistic approach is followed in the context of SCR, where most common events can be categorised as an unknown-unknown risk and where events' probabilities are extremely difficult to obtain. The characteristics of uncertainty elements turn strategic and tactical levels of decision into a fundamental part in the process of building and maintaining a resilient SC (Sáenz, Revilla, and Acero 2018; Sahebjamnia, Torabi, and Mansouri 2015; Cohen and Lee 2020; Kaur and Singh 2019). Thus, macro and network decisions are a crucial factor when handling SCR (Asian and Nie 2014; Kim, Chen, and Linderman 2015; Singh 2020), as it already is in other fields of SCM (Farahani et al. 2013; Mota et al. 2015, 2017).

With SCR appearing as a not yet mature concept, its definition is still a topic of discussion present in the work of different authors. Providing a stable interpretation is crucial to have all the involved parties discussing the issue on the same grounds, a concern recognised by several reviews on SCR that devote some work to its discussion and formalization (Ribeiro and Barbosa-Póvoa 2018b; Kamalahmadi and Parast 2016; Wang et al. 2016; Hohenstein et al. 2015; Tang 2006). In this work, we follow the definition presented by Ribeiro and Barbosa-Póvoa (2018b) where "a resilient supply chain should be able to prepare, respond, and recover from disturbances and afterwards maintain a positive steady-state operation in an acceptable cost and time".

\section{SC resilience: models and metrics}

As in other areas of SCM, quantitative studies have been applied to SCR so as to provide the best insight possible for decision-making. However, due to its particular characteristics, discussed in Sect. 1, its modelling varies in scope, approach and metrics involved.

\subsection{Models}

A straightforward way to think about SCR is establishing what should be done prior and after a disruption (Birkie 2016; Gaonkar and Viswanadham 2007; Han and Shin 2016). It is possible to identify four steps (Prepare, Respond, Recover and Maintain) that are used frequently in the literature with different degrees of description (Hohenstein et al. 2015; Ponis and Koronis 2012; Ponomarov 2012; Kamalahmadi and Parast 2016). Ribeiro and BarbosaPóvoa (2018b) summarise SCR in a mind-map of 4 elements (Focus Event, Performance Level, Speed and Adaptive Framing). It is by combining these different elements that one should consider how to evaluate SCR for each particular case properly. The need for tailored attention to the specification of the focus event and the requirements in terms of speed and adaptive framing lead to a broad set of examples on the SCR metrics used in the literature. The resilience of the whole $\mathrm{SC}$ is the result of the resilience of each element and the interactions between them. The design and planning of SC networks is of extreme relevance since it affects different groups of decision. For instance it will influence inventory costs, production and transportation costs, but it can also imply in what is the growth capability of a company (Song and Sun 2017). Modelling could provide the tools to make better decisions, identifying the weakest links and work with them as best as possible (Adobor 2019).

Moving from acknowledging the core principles of SCR to a requisite model is a challenge. Firstly, one has to generate or acquire a set of elements to be representative of the reality 
to be translated into a mathematical language. The literature provides few examples on how to apply such constructions into models that vastly differ on the Operational Research (OR) method used, and in its scope.

SCR particularities guide authors to follow OR methods that are well-established in the field of SCM, with optimisation being the most frequently used approach, closely followed by simulation or decision analysis (Ribeiro and Barbosa-Póvoa 2018b). While Carvalho et al. (2012) or Schmitt and Singh (2012) face the challenge of designing a resilient SC through a simulation model, Cardoso et al. (2015) explored optimisation, which is recurrently used and recognised as an appropriate method to study the problem of SC design and planning under uncertain conditions, where different objectives are at stake (Ivanov et al. 2017; Ning and You 2018).

\subsection{Metrics}

The most common quantitative resilience approaches rely on maximising only one type of factor, usually financial. On the other hand, the introduction of goals and performance measures is a method used to better describe the model intentions (Barroso, Machado, and Machado 2011; Carvalho, Duarte, and Cruz Machado 2011; Elleuch et al. 2016). Recognizing this, Munoz and Dunbar (2015) quantifies resilience in five dimensions (recovery time, impact, profile length, performance loss and weighted sum). Another approach is the development of a single index, as presented by Azevedo, Carvalho, and Cruz-Machado (2016); Soni, Jain, and Kumar (2014); Wang and Ip (2009); Hosseini, Ivanov, and Dolgui (2019). The latter method leads to a more straightforward and more intuitive way to understand the results and allows a simple comparison between the different cases where the model is to be implemented.

The primary objective of a location problem is to establish a SC network that can fulfil its demand efficiently (Farahani et al. 2014). Being able to meet a pre-established customer service level, even under disruptions, can be a way to study the resilient behaviour of SC (Jahani et al. 2020). Such idea is to be explored in the present work, by combining financial measures with service level leading to a responsive metric for the design and planning of resilient SC.

\section{Problem description}

SC's managers face several challenges when assessing SCR; most of them fall under strategic or tactical SCM decision levels. Decisions on which locations to operate, the quantities to be produced in each location, the outsourcing needs, and the flows to promote between the different possible entities in the network, are to be taken. These should guarantee operations to be cost-efficient while maintaining customer satisfaction (Lotfi and Saghiri 2017). The design and planning of resilient SC are the objects studied in this article.

The most common SC can be depicted in a four-level structure. Starting from Raw Materials and ending in Markets with Plants and Warehouses as intermediate echelons. To entail any possible SC configuration, the problem here studied represents a set of different flows between tiers, from a traditional forward four levels SC to a generic closed-loop SC (CLSC).

Uncertainty is considered in the SC demand and on disruptions, where a scenario tree is constructed, combining the outcomes of these two sources of uncertainty. Demand variability is introduced by generating a scenario for each period from a set of three possible outcomes 
(Pessimistic, Realistic or Optimistic). The disruptions only assume two options, it either occurs, or it does not, in a specific period to consider. The probabilistic binary distribution for the occurrence of disruptions is due to the impracticable definition of probabilities of occurrence of each event (Ivanov and Dolgui 2018), as discussed in Sect. 1. Resulting in a set of scenarios where each scenario probability is given by the probabilities of the path, with all stages between the root node and each leaf node.

\section{Mathematical model}

The model here presented is a strategic-tactical model that supports the design and planning of SC considering a new resilience metric is incorporated to build a resilient SC. This was based on the MILP model developed by Cardoso et al. (2015), here the most relevant elements are presented, and the new model is provided with detail in Annex 1. The main decisions are summarised below and are further detailed in Section 4.1.

- Defining the supply chain network (XNodes $s_{v t}$ - Binary variable equal to 1 if entity $v \in V$ opens in period $t \in T$ and 0 otherwise)

- Defining the flows between entities ( $Y_{v w t}$ - Binary variable equal to 1 if the forward link between entity $v \in V$ and entity $w \in V$ is established at time period $t \in T$ and 0 otherwise; $Y N C_{v w t}$ - binary variable equal to 1 if the reverse link between entity $v \in V$ and entity $w \in V$ is established at time period $t \in T$ and 0 otherwise; $Y E L_{v w t}$-Binary variable equal to 1 if the end-of-life reverse link between entity $v \in V$ and entity $w \in V$ is established at time period $t \in T$ and 0 otherwise)

- Defining the supply chain network, the characteristics of production and the technologies involved ( $i m_{i v}$ - production capacity of technology $i \in I$ in facility $v \in V$ and $C E_{i v t}^{p}$ the capacity expansion of technology $i \in I$, in facility $v \in V$ and in time period $t \in T$ ) and those related with storage $\left(i n_{v}^{s}\right.$ - the initial capacity of facility $v \in V$ and $C E_{v t}^{s}$ - the expansion of storage capacity of facility $v \in V$ in time period $t \in T$ )

Consider the following sets:

$$
\begin{aligned}
& \quad V \text {, set of entities/locations } \\
& V_{w h} \subseteq V \text {, set of entities with warehousing } \\
& V_{m} \subseteq V \text {, set of markets } \\
& V_{g} \subseteq V \text {, set of suppliers } \\
& V_{c} \subseteq V \text {, set of entities with technologies } \\
& F_{v, w}, \text { set of pairs }(v, w) \text { such that a forward flow is allowed between } v \text { and } \\
& \quad w, v, w \in V \\
& R_{v, w}, \text { set of pairs }(v, w) \text { such that a reverse flow is allowed between } v \text { and } \\
& \quad w, v, w \in V \\
& \quad S, \text { set of nodes in scenario tree } \\
& P, \text { set of products } \\
& P_{f} \subseteq P \text {, set of final products } \\
& I, \text { set of available technologies } \\
& T, \text { set of time periods }
\end{aligned}
$$


This work focuses on the definition of a new SCR metric, which aims to account not only for economic return but also for customer service level.

\subsection{SC management key performance indicators}

A set of SCM performance metrics is here considered to express SCR and can be used for a more in-depth analysis of the problem. This is based on commonly used network design resilience metrics. Flow complexity adds flexibility and redundancy to the SC, being already proved to be a very good indicator of SCR (Cardoso et al. 2015). These strategies are, however, far from being feasible "plug and play" solutions in real applications (Ribeiro and Barbosa-Póvoa 2018a). For instance, back-up suppliers might lead to performance degradation if the SC is still reliant on unreliable suppliers(Demirel, Kapuscinski, and Yu 2018). Two operational metrics are often used: Expected Net Present Value (ENPV) and Expected Customer Service Level (ECSL) have been shown to drive supply chain management. It is natural for a new metric to emerge, combining the two operational indicators aiming to be a more comprehensive SCR metric. In this new metric, both economic and responsiveness goals of the supply chain are achieved while guaranteeing supply chain resilience. Although the forehand listed metrics can be present as resilience metrics, it is impossible to retrieve any conclusion regarding the resilience of a given SC solely from an individual parameter. Thus, these metrics must be used in combination and relative to each other to provide useful information.

The mentioned base metrics are modelled as follows:

\subsubsection{Flow complexity}

Total quantity of flows between entities, including forward flows (Y), reverse flows of nonconforming products (YNC) and the reverse flows of end of life products (YEL);

$$
F C_{t}=\sum_{v \in V} \sum_{w \in V}\left(Y_{v w t}+Y N C_{v w t}+Y E L_{v w t}\right) \quad \forall t \in T
$$

\subsubsection{ENPV}

The Expected Net Present Value (€) is calculated for each scenario and period, considering cash flows $\left(C F_{s t}\right)$ and an interest rate (ir), Eq. (2);

$$
\begin{aligned}
N P V_{s} & =\sum_{t \in T} \frac{C F_{s t}}{(1+i r)^{t}} \quad \forall s \in S \\
C F_{s t} & =N E_{s t}-F D C_{t} \quad t=1, \ldots,|T|-1, \quad \forall s \in S
\end{aligned}
$$

The cash flows are given by Eq. (3) represented by the difference between the net earning and the fraction of the depreciated capital. Net earnings $\left(N E_{s t}\right)$, Eq. (4), come as result of the difference between the income $\left(\mathrm{Eg}\right.$. Sales revenue $\left(S_{v p s t}\right)$ and the refunds from non-conforming products $\left.\left(R Q N C_{v w p s t}\right)\right)$ and the costs (Eg.: purchasing products $\left(P u_{\text {pvst }}\right)$, operational $\left(O p_{\text {ivpst }}\right)$, inventory $\left(I C_{v s t}\right)$, transportation $\left(T C_{v w t}\right)$ and depreciation of invested capital $\left(D P_{t}\right)$ considering taxes $(t r) .|T|$ stands for the number of time periods involved in the model; 


$$
\begin{aligned}
N E_{s t}= & (1-t r)\left[\sum_{v \in V_{m}} \sum_{p \in P} S_{v p s t}-\sum_{v \in V_{m}, w \in V:(v, w) \in R_{v, w}} \sum_{p \in P} R Q N C_{v w p s t}\right. \\
& \left.-\sum_{v \in V_{g}} \sum_{p \in P} P u_{p v s t}-\sum_{i \in I} \sum_{v \in V} \sum_{p \in P} O p_{i v p s t}-\sum_{v \in V_{w h}} I C_{v s t}-\sum_{v \in V} \sum_{w \in V} T C_{v w t}\right] \\
& +\left(t r \times D P_{t}\right) \quad \forall t \in T \quad \forall s \in S
\end{aligned}
$$

\subsubsection{ECSL}

Expected Customer Service Level (\%) is the percentage of demand met, as the relationship between the unsatisfied demand $\left(\sum_{v \in V_{m}} \sum_{p \in P} I D_{p v s t}\right)$ and the total demand $\left(\sum_{v \in V_{m}} \sum_{p \in P} D_{p v s t}\right)$. Unsatisfied demand has to be calculated as a result of the decisions made during the time periods that influence the difference between the actual demand and the flows sent to each market $\left(\sum_{v \in V_{m}, w \in V:(v, w) \in F_{v, w}} Q_{w v p s t}\right)$;

$$
E S C=\frac{\sum_{t \in T}\left(1-\sum_{s \in S} p b_{s}\left[\frac{\sum_{v \in V_{m}} \sum_{p \in P} I D_{p v s t}}{\sum_{v \in V_{m}} \sum_{p \in P} D_{p v s t}}\right]\right)}{|T|}
$$

Unsatisfied demand is given by Equation 6, representing the difference between the demand recognised for each market and product, for each scenario and period $\left(D_{p v s t}\right)$, and the quantity of product delivered to such market $\left(\sum_{w:(w, v) \in F} Q_{w v p s t}\right)$. $P_{f}$ represents the set of final products and $S$ represents the nodes of the scenario tree;

$$
I D_{\text {pvst }}=D_{\text {pvst }}-\sum_{v \in V_{m}, w \in V:(v, w) \in F_{v, w}} Q_{w v p s t} \quad \forall p \in P_{f} \wedge v \in V_{m} \wedge \forall t \in T \forall s \in S
$$

\subsubsection{Auxiliary variables}

- FCI (€) Fixed Capital Investment is the summation of costs associated with strategic decisions, in which each parcel represents different investments: the occurrence of each process $\left(\sum_{i \in I} \sum_{v \in V} i m_{i v} \times i n_{i v}^{p}\right)$, the eventual expansion of processes capacity $\left(\sum_{i \in I} \sum_{v \in V_{c}} \sum_{t \in T}\left(v_{i v t}^{p} \times C E_{i v t}^{p}\right)\right)$, the cost of storage entities $\left(\sum_{v \in V} i s_{v} \times i n_{v}^{s}\right)$, the eventual storage expansion $\left(\sum_{v \in V_{w h}} \sum_{t \in T}\left(v_{v t}^{s} \times C E_{v t}^{s}\right)\right)$ and the investment needed to implement new links between entities $\left(\sum_{v \in V} \sum_{w \in V} \sum_{t \in T} l k_{v w t} \times\left(Y_{v w t}+Y N C_{v w t}+\right.\right.$ $\left.Y E L_{v w t}\right)$

$$
\begin{aligned}
F C I= & \sum_{i \in I} \sum_{v \in V} i m_{i v} \times i n_{i v}^{p}+\sum_{i \in I} \sum_{v \in V_{c}} \sum_{t \in T}\left(v_{i v t}^{p} \times C E_{i v t}^{p}\right) \\
& +\sum_{v \in V} i s_{v} \times i n_{v}^{s}+\sum_{v \in V_{w h}} \sum_{t \in T}\left(v_{v t}^{s} \times C E_{v t}^{s}\right) \\
& +\sum_{v \in V} \sum_{w \in V} \sum_{t \in T} l k_{v w t} \times\left(Y_{v w t}+Y N C_{v w t}+Y E L_{v w t}\right.
\end{aligned}
$$


Where:

$$
\begin{aligned}
& \text { im } m_{i, v} \text { Capacity of technology } i \in I \text { in entity } v \in V \\
& \text { in } n_{i, v}^{p} \text { Initial investment associated with each technology } i \in I \text { in each } \\
& \text { entity } v \in V \\
& C E_{i, v, t}^{p} \text { Technology } i \in I \text { capacity expansion in entity } v \in V \text { over time } \\
& \text { period } t \in T \\
& v_{i, v, t}^{p} \text { variable investment associated with each technology } i \in I \text { in each } \\
& \text { entity } v \in V \text { in each time period } t \in T \text { in euros } \\
& \text { in } n_{v}^{s} \text { initial capacity of each entity with storage, } v \in V_{w h} \\
& \text { is } s_{v} \text { initial investment associated with each entity with storage, } v \in V_{w h} \\
& C E_{v, t}^{s} \text { Storage expansion of entity } v \in V \text { over time period } t \in T \\
& v_{v, t}^{s} \text { variable investment associated with each entity } v \in V \text { with storage } \\
& \text { in time period } t \in T \text { in euros } \\
& l k_{v, w, t} \text { contract cost of a carrier to make a journey from one entity } v \in V \\
& \text { to another } w \in V \text { in the period } t \in T \text { in euros }
\end{aligned}
$$

- Investment $(€)$ The total investment in the SC infrastructure, not including transportation costs;

$$
\begin{aligned}
\text { Investment }= & \sum_{i \in I} \sum_{v \in V} i m_{i v} \times i n_{i v}^{p} \\
& +\sum_{i \in I} \sum_{v \in V_{c}} \sum_{t \in T}\left(v_{i v t}^{p} \times C E_{i v t}^{p}\right)+\sum_{v \in V} i s_{v} \\
& \times i n_{v}^{s}+\sum_{v \in V_{w h}} \sum_{t \in T}\left(v_{v t}^{s} \times C E_{v t}^{s}\right)
\end{aligned}
$$

- Inventory $(€)$ Inventory present in warehouses is represented in monetary terms taking into account the probability of all scenarios $\left(p b_{s}\right)$. The unitary cost of holding inventory $\left(C i n v_{v t}\right)$ and the amount of inventory present in each entity at each scenario for each time period $\left(I L_{v s t}\right)$ are considered;

$$
\text { Inventory } y_{t}=\sum_{s \in S} \sum_{v \in V_{c}}\left(p b_{s} \times C i n v_{v t} \times I L_{v s t}\right) \quad \forall t \in T
$$

- Purchases (€) Purchases at time $t$ are represented taking into account the probability of all scenarios $\left(p b_{s}\right)$, the unitary cost of acquiring a certain good $\left(\mathrm{rm}_{p v t}\right)$ and the amount of products bought in each entity at each scenario for each period $\left(P u_{p v s t}\right)$;

$$
\text { Purchases }_{t}=\sum_{s \in S} \sum_{v \in V_{g}} \sum_{p \in P} p b_{s} \times r m_{p v t} \times P u_{p v s t} \quad \forall t \in T
$$

- Sales $(€)$ Total sales are given by the price of products ( Price $_{p v t}$ ) and the amount of goods sold $\left(S a_{\text {pvst }}\right)$;

$$
\text { Sales }_{t}=\sum_{s \in S} \sum_{v \in V_{m}} \sum_{p \in P} p b_{s} \times \text { SPrice }_{p v t} \times S a_{p v s t} \quad \forall t \in T
$$




\subsection{New SC resilience performance metric}

As identified by Ribeiro and Barbosa-Póvoa (2018b), Wang et al. (2016), Kamalahmadi and Parast (2016), Hohenstein et al. (2015) there is a need to develop more comprehensive SCR metrics. In this work, we pursue this goal and a SCR metric involving economic goals and service level is developed. This is considered in the model as an objective function (OF) and is defined as follows in Eq. (12).

$$
\operatorname{Max} \frac{\sum_{s \in S} p b_{s} \times N P V_{s}}{N P V_{\text {ref }}}-\frac{\sum_{s \in S} p b_{S}\left[\frac{\sum_{v \in V_{m}} \sum_{p \in P} I D_{p v s t}}{\sum_{v \in V_{m}} \sum_{p \in P} D_{p v s t}}\right]}{|T|}
$$

The first term represents the economic concern modulated through a variation rate between the resilient SC ENPV and the ENPV obtained for the SC network that maximises ENPV when no disruption occurs $N P V_{\text {ref }}$. This term will vary, for any positive number smaller than one if ENPV values are lower than the reference. However, it is not expected for this value to go above the unitary value since the $N P V_{\text {ref }}$ is the best economic performance when compared to a situation where disruptions occur. Generically, this parcel is responsible for penalising deviations from the best ENPV possible, with the values closest to one being those that most positively impact SC performance.

The second term, introduces the concern of meeting customer demand as a key decision factor when a strategic decision is to be made. This term assumes values between zero and one, as it represents the mean value for the fraction between the unsatisfied demand and total demand. Generically, this parcel is responsible for not letting the customer service level drop in complete favour of economic return and will generate the most benefit to the $\mathrm{OF}$ as close to zero as possible.

The relationship between the two parcels implies that the model will value the increase of ENPV equally as the decrease of unsatisfied demand, implying that the percentage variations are equally relevant to the decision. The definition of monetary values for non-satisfied demand introduce deviations that change from case to case and can be challenging to obtain when considering a longer time span, as is this case. Defining a target service level, in the context of disruptions can also mislead results, due to its relationship with steady-state and disruptions. Therefore, in the context of measuring the resilient behaviour of a SC, this approach returns appropriate information to decision-makers on how will the SC respond to disruptive scenarios and easily allows for comparisons between different SC.

\subsection{Model main constraints}

As the design and planning of resilient supply chains are at stake, it is crucial to consider different problem characteristics that arise when focusing on generic supply chain networks. This involves a set of constraints as: mass balances; demand levels; capacity restrictions; flow definition; transportation links. This are described in detail in Annex 1.

\section{Disruptive scenarios}

The quest for the identification of events that cause disruptions in SC is not new. Most authors identify representative episodes that could be implemented in models. The results of the COVID-19 pandemic shows us that this is a sound approach, since most events that 
occurred could have been prepared following such strategy. Works already published are consistent with such idea (Azad et al. 2013; Elluru et al. 2019), most retaining information from pandemic case studies. Hobbs (2020) identified two types of disruptions for the Canadian food industry, the lack of raw materials and transportation disruptions brought by the restrictions on movements of goods and people. Production and raw materials can be affected, as there was a global effort to maximise production on essential items, as toilet paper or medical provisions, that can lead to stoppages in production of the usual items (Paul and Chowdhury 2020).

In order to properly understand how the results of the new metric and the presented model impact on the SCR, three failure modes are identified (Production, Supply, Transportation), adapted from (Rice and Caniato 2003), and are to be studied.

\section{- Disruption 1 production facilities}

This disruption is representative of all the failures that can cause factories to cease production. The COVID-19 pandemic showed us several examples of this. Some, due to imposed lock-down which in consequence forced factories to stop. Some parts of Italy closed theirs factories in an effort to contain the outbreak (RTE 2020). In the area of Milan, the mandatory shutdown lasted for months and the return to normal was done gradually, depending on the type of service (Jewkes and Amante 2020).

Production could also stop if for some reason there was no workforce available. The traditional example of labour strikes had similar consequences to factories that had to stop operations due to infections of SARS-COV-2 amongst their workers. This forced stop could happen in all kinds of factories, since regardless of the relevance of the product being made, keep on working could pile up infections inside and outside the factory. An example of this occured in a meat processing plant in the United States (US), that was forced to close even after initial efforts to remain open due to its relevance in food production (Lussenhop 2020).

- Disruption 2 Supply

Shortages of raw materials represent a fairly common event in SC. The COVID-19 pandemic brought it to a completely different level. This type of events were fairly brief, in contrast to recent shortages that lasted for months. This was particular evident in the pharmaceutical industry, with raw materials distribution halted which in turn lead to shortages of medical drugs (Mullin 2020; Fitch Solutions 2020).

- Disruption 3 Transportation Even for industries that were able to keep working and producing some had a new hurdle, the transportation of goods to their final destination. Many countries prohibited non-essential movements, most often international ones. In some cases it also included transportation of goods (air 2020). Even traditionaly interconnected countries had their exchanges halted as was the case of the border between the US and Canada (Hobbs 2020). Who would ever thought that the Schengen space would close completely in such a small period of time (Wimmer 2020)? Thus, transportation disruptions are representative and give an insight into how prepared a SC must be to be resilient.

With this interpretation for the generation of a representative set of disruptions, previously used and presented in the literature, it is possible to establish a simple and replicable methodology not only for comparison with previous publications but also for new developments in the field of SCR. It enables the study of the resilient behaviour of the SC while providing relevant data, facilitating the improvement of the responsiveness of the SC while easing the dissemination of managerial insights for practitioners and academics alike. 


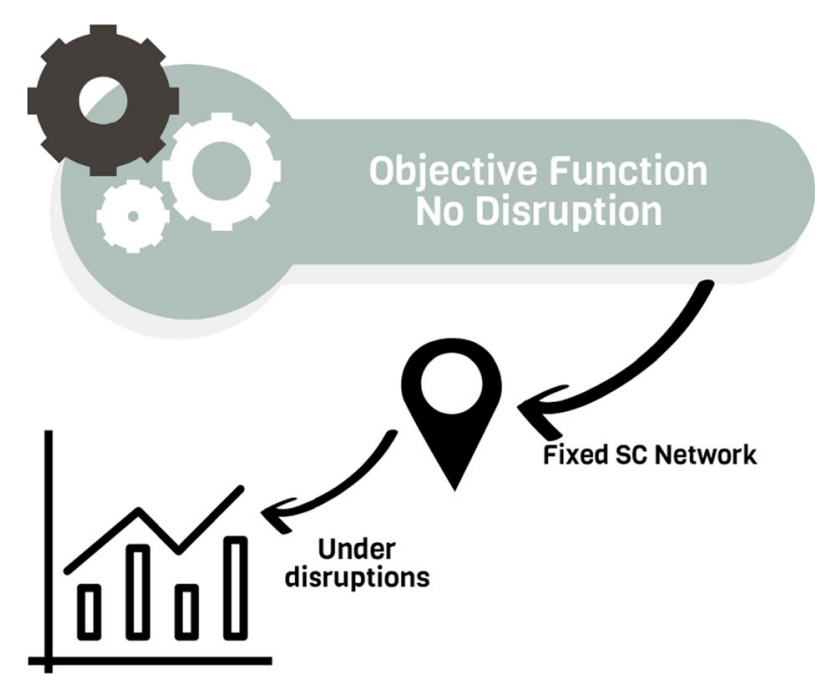

Fig. 1 Heuristic for acquiring results

\section{Maximising SC ENPV versus maximising SCR performance}

For a comprehensive and valid process of results acquisition and discussion, a structured methodology is followed, represented schematically in Fig. 1. For each SC case, defined later on in Sect. 6.2, the network configuration is established based on the SC network provided by the objective function when no disruption occurs. The obtained network design is then tested under a disruption scenario, as characterised in Sect. 5, and the SCR results are analysed and discussed.

The MILP model is implemented in GAMS software and solved with CPLEX.

With this study, it is possible to compare the SCR in terms of different metrics and understand the relevance of the new proposed SCR metric and its impact on the SC network design.

\subsection{Case study description}

The presented model is tested in a case study. This case study is based on a European SC with the associated data scaled down due to confidentiality reasons.

To improve SC's capacity to withstand disruptive events, while expanding its business, the company is studying several possibilities and acquiring the best information possible to make an informed decision. In broad terms, the company is willing to reconfigure the existent SC by upgrading the technologies used on the current entities or invest in new entities; Plants, Suppliers or Warehouses.

As mentioned, the data is modified to respect company confidentiality, but this does not affect the SC characteristics.

\subsubsection{Supply chain characteristics}

The initial SC is supplying 17 European markets. Final products can be manufactured in a plant in Hamburg or be bought in outsourcing suppliers located in Riga, Minsk and Warsaw. When produced in-house, the SC relies on raw materials sourced from suppliers in Frankfurt, 

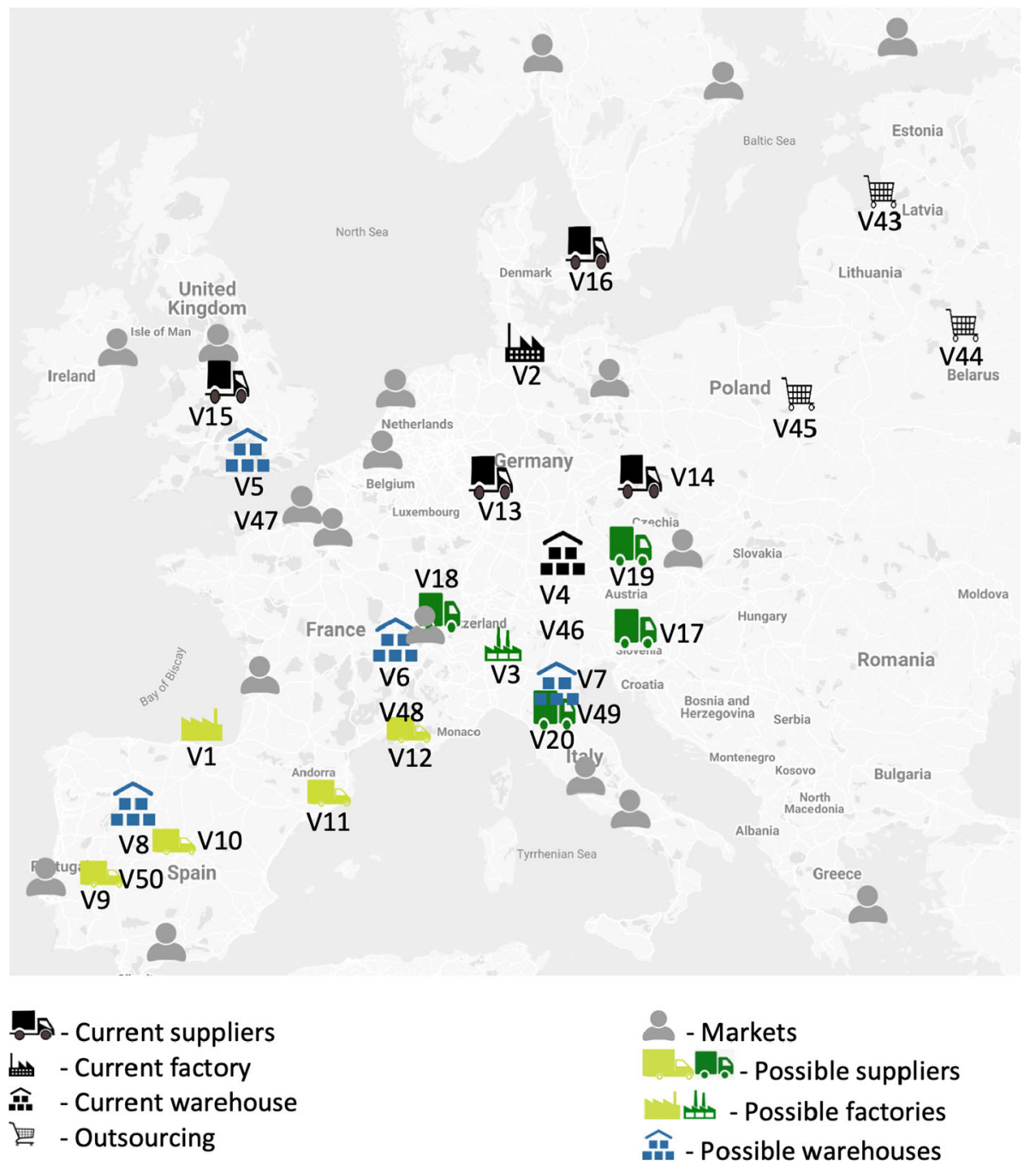

Fig. 2 SC network configuration with current and prospective facilities

Prague, Birmingham and Copenhagen. The raw materials are then brought to the plant where twelve production technologies and six disassembling technologies are used in the production of products. The latter technologies are allocated to the reserve flows of used products, if existing. There is an intermediate echelon constituted by one warehouse in Munich, with a capacity of 500 units, that creates value not only by managing the inventory but also by being able to assemble goods with six technologies.

With an expansion strategy, the SC is considering the possibility of including two new plants with the following set of raw material suppliers:

- Bilbao

- Badajoz, Toledo, Barcelona, Marseille 
Table 1 Case study costs table

\begin{tabular}{ll}
\hline Costs & \\
\hline Inventory costs & $0.3 € /$ ton period \\
Transportation costs & \\
Fixed costs & $200 € /$ forward transportation link \\
& $150 € /$ reverse transportation link \\
Variable cost & $0.1 € /$ t km for forward flow \\
& $0.2 € /$ t km for reverse flow \\
Outsourced final production costs per & $p_{29}=250 €, p_{30}=270 €, p_{31}=240 €$, \\
ton for each product $p_{x}$ & $p_{32}=290 €, p_{33}=255 €, p_{34}=265 €$ \\
Plants' investment cost & $1 € /$ ton \\
Warehouses' investment cost & $0.5 € /$ ton \\
Disassembling centres' investment & $0.1 € /$ ton \\
cost &
\end{tabular}

- Milan

○ Ljubljana, Lausanne, Linz, Florence

The prospective warehouse locations are Portsmouth, Lyon, Bologna and Salamanca. The possible structure of the SC considered in the case study is represented in Fig. 2.

Production of products is associated with the implementation of technologies in each plant. Raw materials and intermediate products are also considered. The activities associated with reverse logistics are associated with Warehouses in the case of assembling or re-producing the final products. In plants, there is the case of disassembling end of life products allowing the salvage of products, and the exploration of circular economy concepts.

The costs associated with the case study are expressed in Table 1:

A set of relevant restrictions and conditions also exist and must be considered.

- Capacity expansions lower than 400000 ton/entity

- Flow between entities lower than 5000 ton

- Warehouse turnover ratio is equal to 20 , and no initial inventories are considered

- $20 \%$ of products sent to markets each period are non-conforming

- A minimum of $10 \%$ of products to be collected at markets, $20 \%$ of the collected goods are sent to disposal

- Demand is assumed to be known for the first period. For the second period, the optimistic scenario has a probability of 0.25 with an increased demand of $10 \%$, the realistic scenario with a probability of 0.5 and an increase of $3 \%$ and finally, the pessimistic scenario has a probability of 0.25 causing a decrease in demand of $2 \%$. In the third period, the scenarios have the same probability as in the second period, but with different effects on the demand, the optimistic causes an increased demand of 5\%, the realistic $2 \%$ and finally the pessimistic causing a decrease in demand of $2 \%$

- Interest rate $=10 \%$, Salvage Rate $=20 \%$, Tax Rate $=30 \%$

- All costs are penalised by $5 \%$ in the subsequent periods of time 


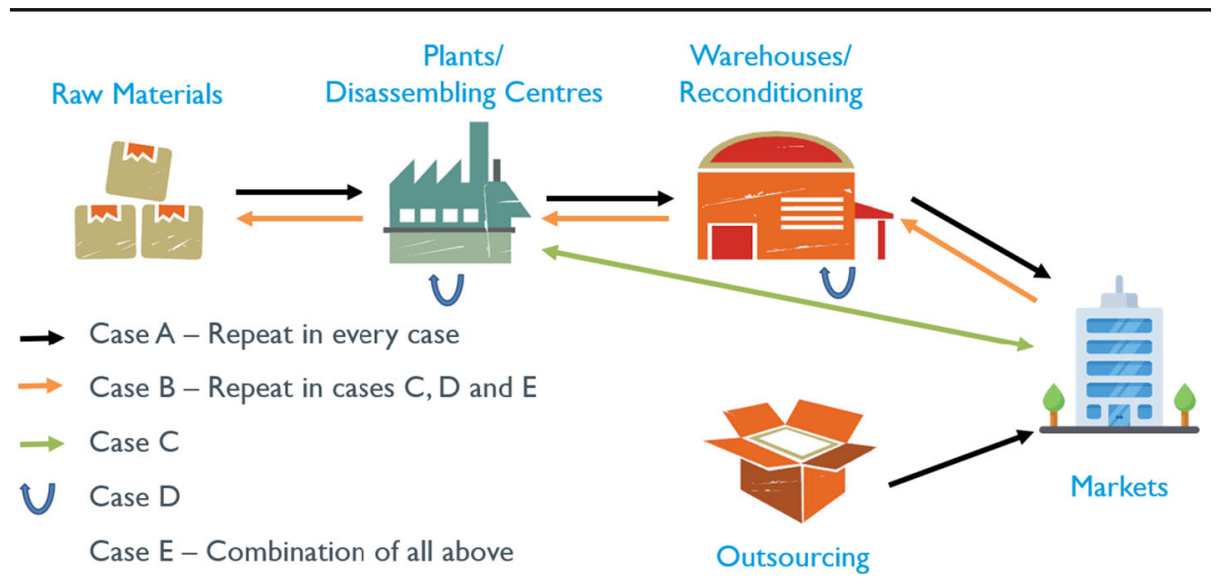

Fig. 3 Conceptual description of the SC network, with all cases considered

\subsection{Possible SC network configurations}

Five different cases, with different SC possible network configurations, under steady-state conditions (no disruption) and a set of disruptive scenarios are analysed. The comparison between the combination of the various cases and disruptive scenarios helps to identify how the SC network can be designed to achieve higher resilience. The explored cases are depicted in Fig. 3, allowing for the generation of comparable results.

- Case A a forward supply chain;

- Case $B$ forward and reverse flows between consecutive echelons;

- Case $C$ forward and reverse flows between consecutive echelons, but plants and markets can directly exchange products, thus bypassing the warehouses;

- Case D forward and reverse flows between consecutive echelons, but with the possibility of transhipment at plants, disassembling centres and warehouses;

- Case E A closed-loop supply chain where all flows previously described are possible.

\subsubsection{Disruptive events: case study}

The SC is to be evaluated under four operational conditions; one reference case with perfect operational conditions and three types of disruptions. From the discussion in Sect. 5 the representative set of disruptions is applied to the case study. One event for each type of disruption is created and is believed to be a good approach for the study of SCR. The disruptive events are defined below.

- Steady-state No disruptions occur

- Disruption 1 (Production Facilities)- The plant located in Milan, which is the one with higher production, ceases production in time period 2 ;

- Disruption 2 (Supply)- The raw materials suppliers, with higher contributions, located in Birmingham, Badajoz, Marseille, Ljubljana and Lausanne cannot supply in time period 2;

- Disruption 3 (Transportation)- The plant in Milan, which is the one with higher production, cannot send any product to warehouses in Munich, Portsmouth and Bologna in time period 2. 


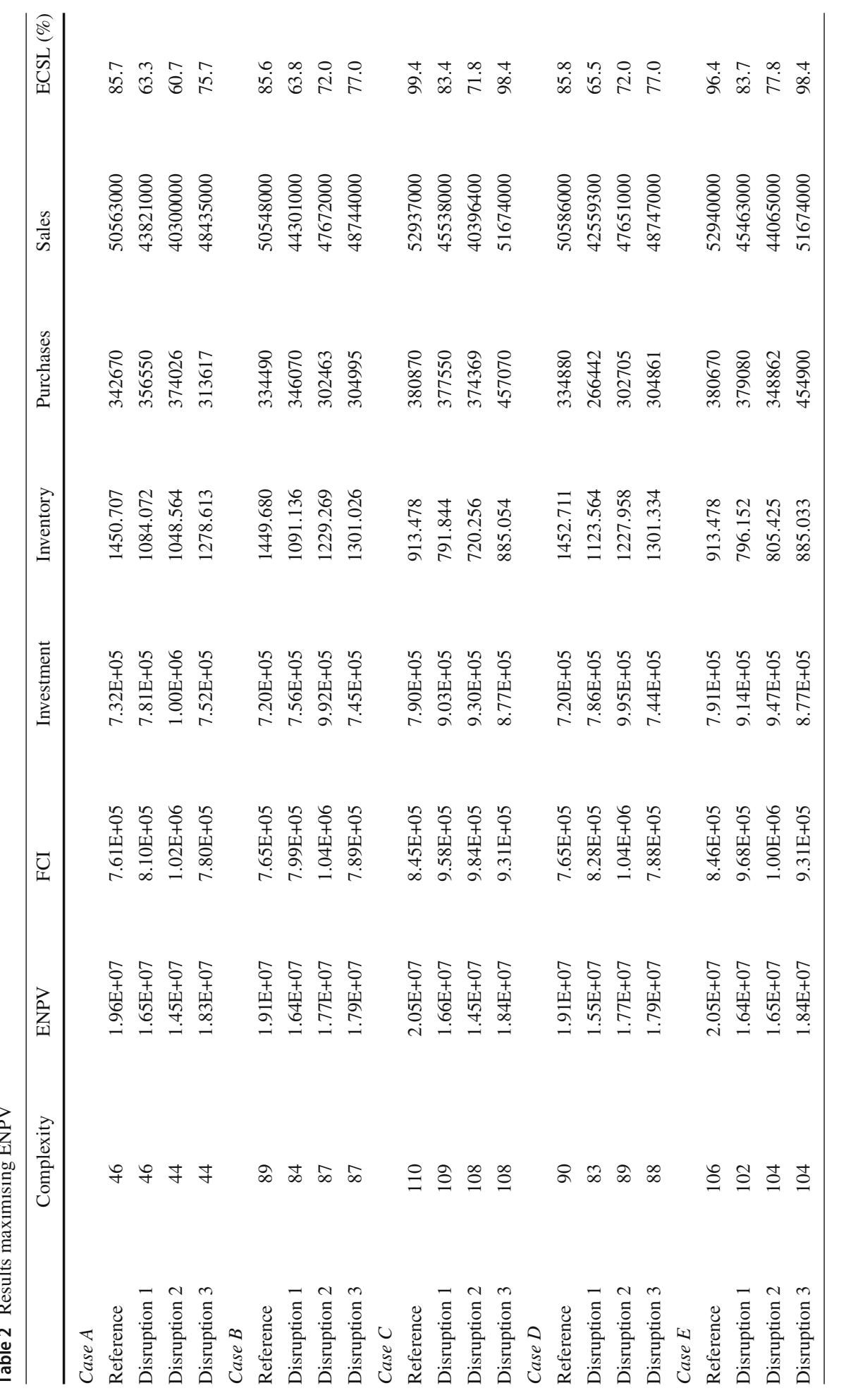




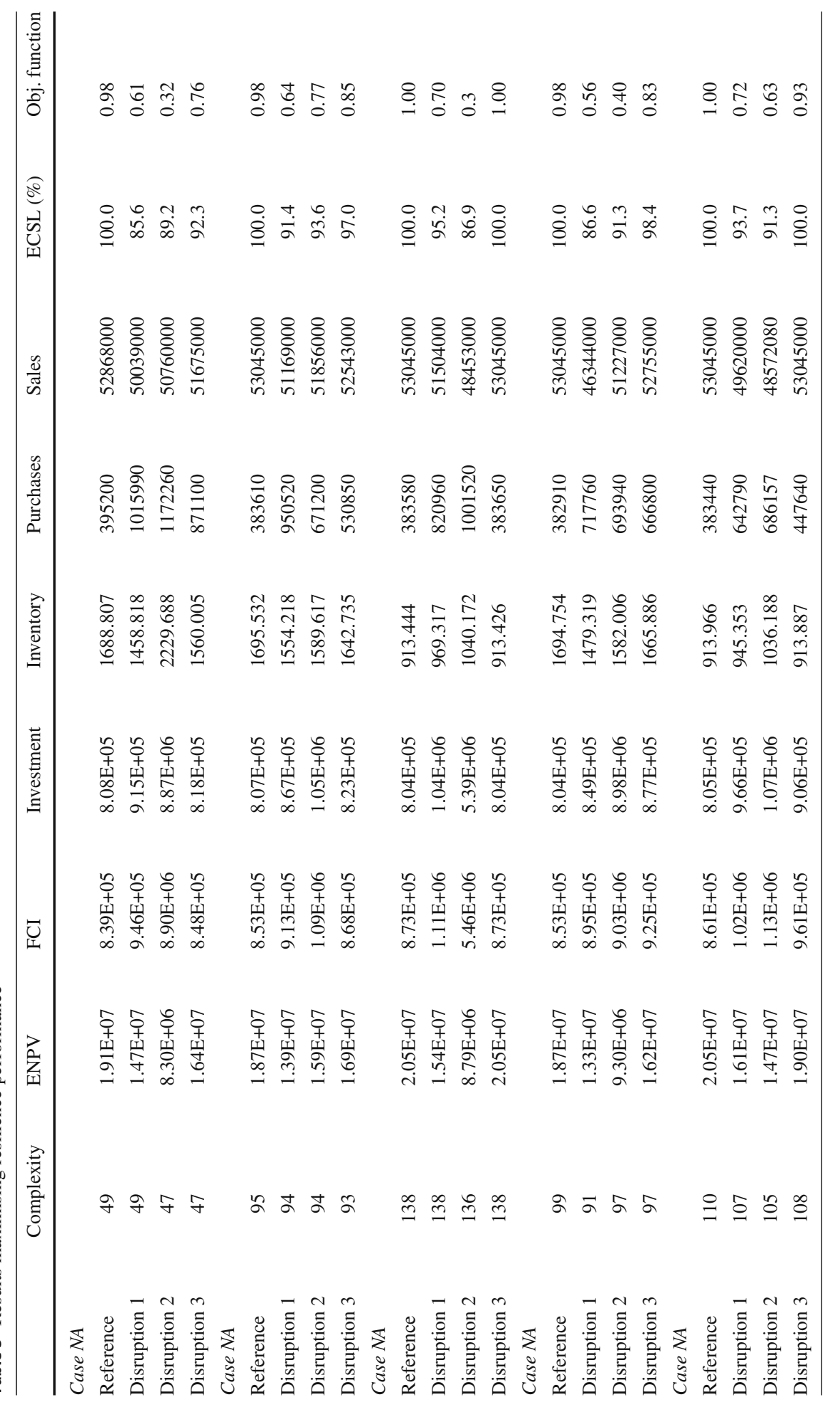




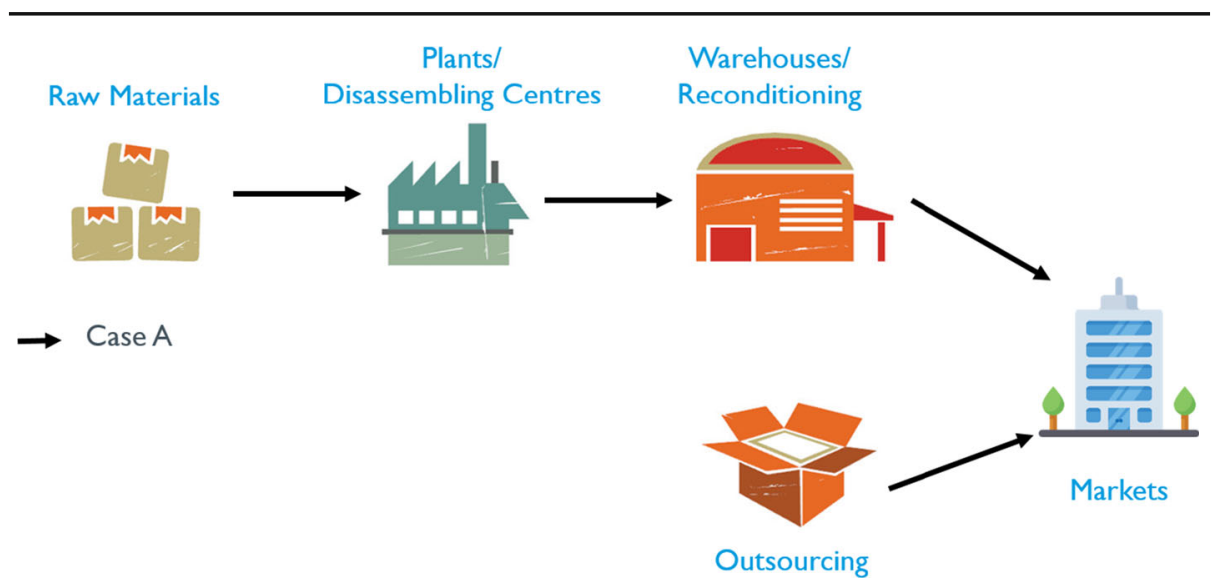

Fig. 4 Schematic configuration of flows allowed in SC Network Configuration A

\subsection{Results}

The case study was applied with the presented model optimising the contrasting metrics here discussed; maximising ENPV or the new resilience metric. The cases related with the maximisation of ENPV are described as "Case $x$ " while those related with the new SCR metric are described as "Case $N x$ ".

On the one hand, the supply chain performance behaviour is presented, Sect. 6.3.1, focusing on SCM performance indicators, allowing for a broader comparison between the two models. Those indicators vary from SC network characteristics (SC complexity, a usual resilience indicator) to economic and operational SC characteristics. On the other, a more in-depth analysis of the ripple effect is performed in Sect. 6.3.2. This explores a better understanding of how could the new metric improve SC responsiveness and resilience. Additionally, the visualisation of flows is presented, allowing for a comparison between different instances and recognise differences in fundamental decisions of SCM.

\subsubsection{Supply chain performance}

Following the methodology described in Section 6, the results when maximising SC ENPV are presented in Table 2 and results from the maximisation of SCR performance, based on the newly proposed metric, are presented in Table 3. Comparing the results in both tables is clear that the new metric optimisation leads to a more resilient SC, with increased responsiveness, enabling to handle disruptive events better. In detail, each case is analysed below.

Supply chain network configuration A Case A represents a simple SC structure (lower network complexity), allowing only forward flows, as depicted in Fig. 4. For the maximisation of ENPV, and from Table 2 it can be seen that, under disruptions, the network loses some of its capacity to meet customer demand (ECSL) and consequently reduces its financial performance (ENPV).

On the other hand, when considering the new SCR metric, the results obtained are substantially different, Table 3. Generically, the results show a lower value in ENPV and a higher value for the expected customer service level (ECSL). Such behaviour translates the increased guarantee of customer satisfaction at the expense of lower profits. 


\section{Case A $\quad \cdots . .$. Case NA}

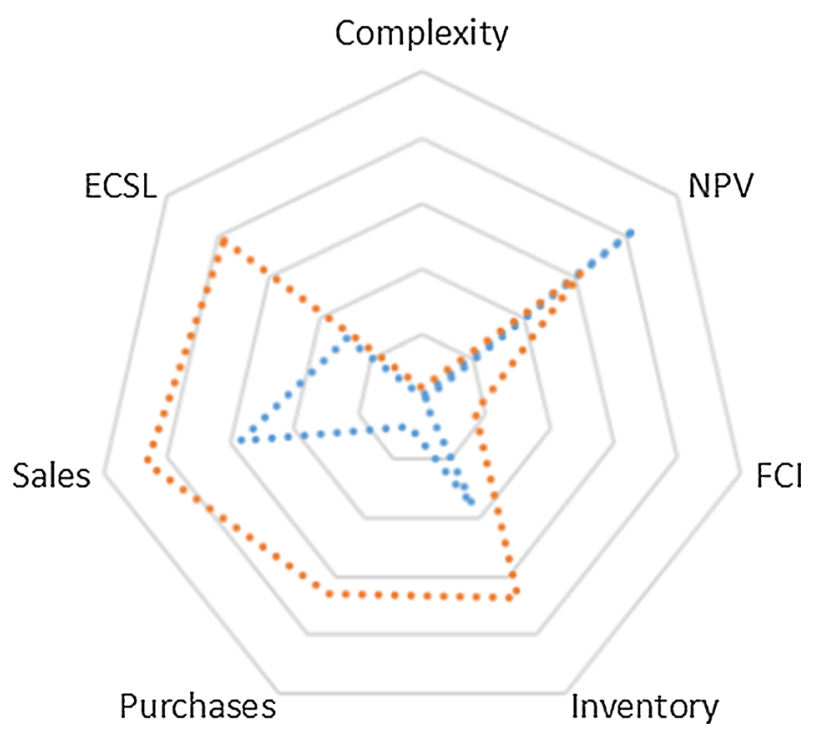

Fig. 5 Average results of SC network configuration A for the two presented models

In the eventuality of no disruption occurring, the OF value in Table 3, stays close to 1, revealing the similarity between the network configuration from the Reference case when maximising the ENPV (Table 2) and the new SC network configuration. The new metric provides a slightly smaller return in ENPV, 1.91 versus 1.96, however, the ECSL moves from 85.7 to $100 \%$.

From Table 3, Case NA, it can be seen that between disruptions there are significant ENPV fluctuations. Disruption 2 returns the lowest ENPV amongst the different disturbances, also leading to a lower value of the presented resilience metric. In turn, Disruption 3 returns the higher value of ENPV and with the smallest percentual variation to the results from ENPV maximisation, $10 \%$. Other disruptions also negatively affect the ENPV, with reductions of $11 \%$ and $53 \%$ for Disruptions 1 and 2, respectively.

With the proposed new OF, there is an innovative relevance being given to ECSL that does not exist when maximising ENPV. This new element brings a significant improvement in service quality, leading to an ECSL of above $85 \%$ for all disruptions. The latter value is better than that returned by the reference model, where the mean ECSL for Case A is $70.37 \%$. Comparatively, there are deviations of $35 \%, 24 \%$ and $22 \%$ for Disruptions 1,2 and 3 respectively. This effort to meet customer demand does not come from added complexity but mainly from an increased number of purchases of final products from outsourcing, which allows for an increase in sales and consequently in ECSL, visible in Fig. 5 where the normalised average results from each model are presented.

Supply chain network configuration B Case B represents a SC structure where reverse flows are allowed between consecutive echelons adding complexity to the operation, Fig. 6.

For this structure, when maximising ENPV, disruptions cause negative impacts on several performance metrics, particularly on the ECSL, with decreases higher than $10 \%$ when compared with the reference state operation. 


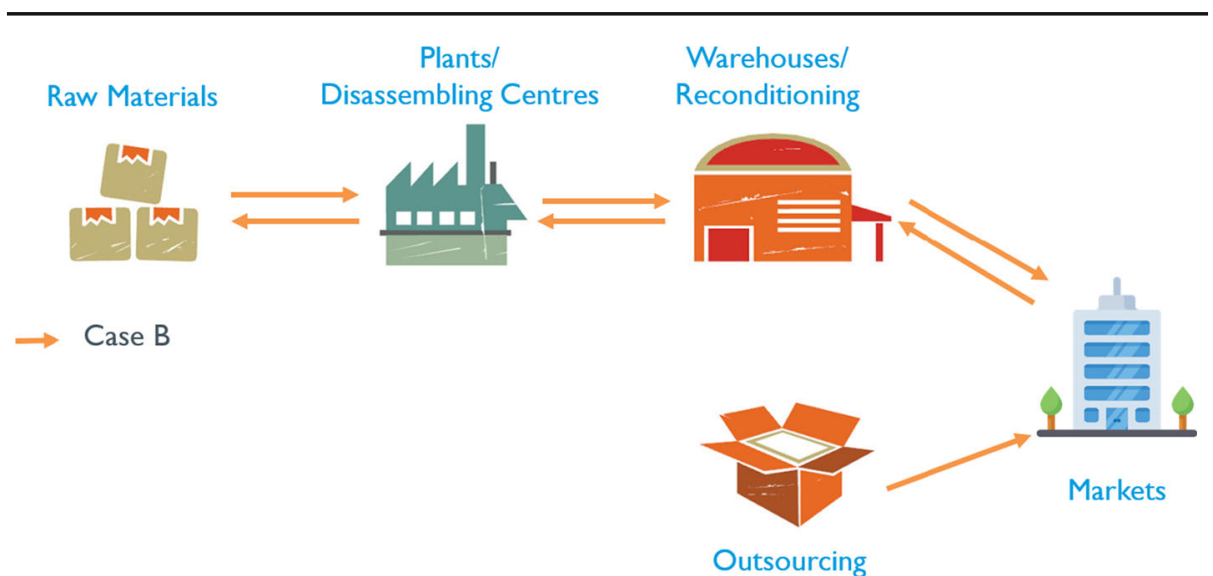

Fig. 6 Schematic configuration of flows allowed in SC Network Configuration B

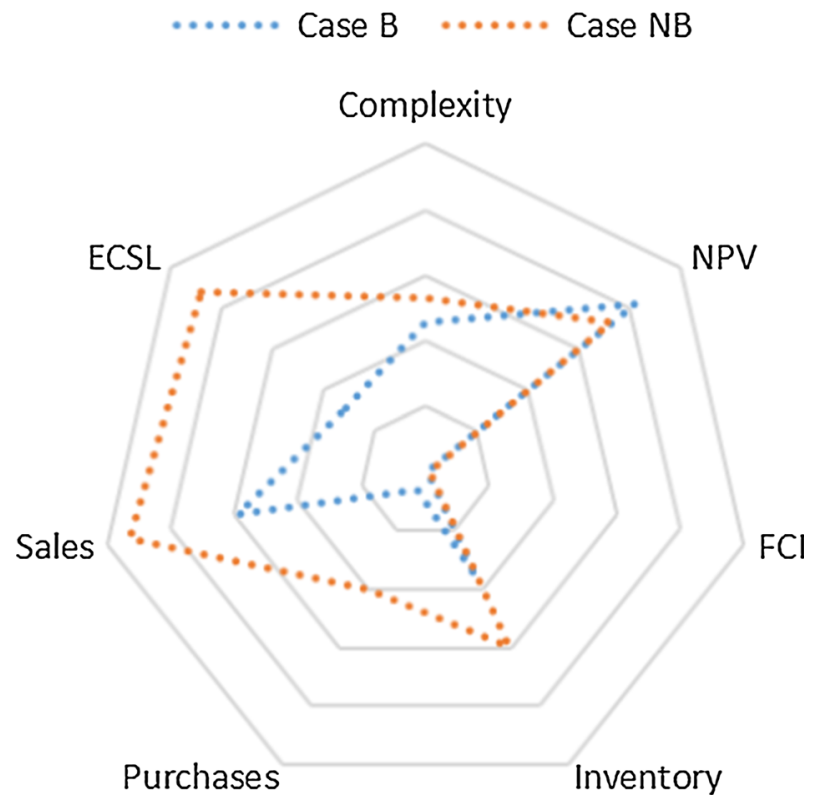

Fig. 7 Average results of SC network configuration B for the two presented models

When maximising the new resilience performance metric, ENPV values are expected to be lower than those from the reference configuration to all cases, due to the inclusion of customer demand satisfaction in the OF. Disruption 1 returns the lowest value and Disruption 3 the higher for the new SC network configuration. These decreases in value represent a variation of $-15 \%,-10 \%$ and $-6 \%$ for Disruptions 1,2 and 3 respectively, when compared to the reference results.

Nevertheless, there is a significant increase in the SC responsiveness, with ECSL being always above $90 \%$, even during disruptions, representing values higher than those provided by the original model when no disruption was present. Comparatively, it produces better results than the economically driven model by $43 \%, 30 \%$ and $26 \%$ for Disruptions 1, 2 and 


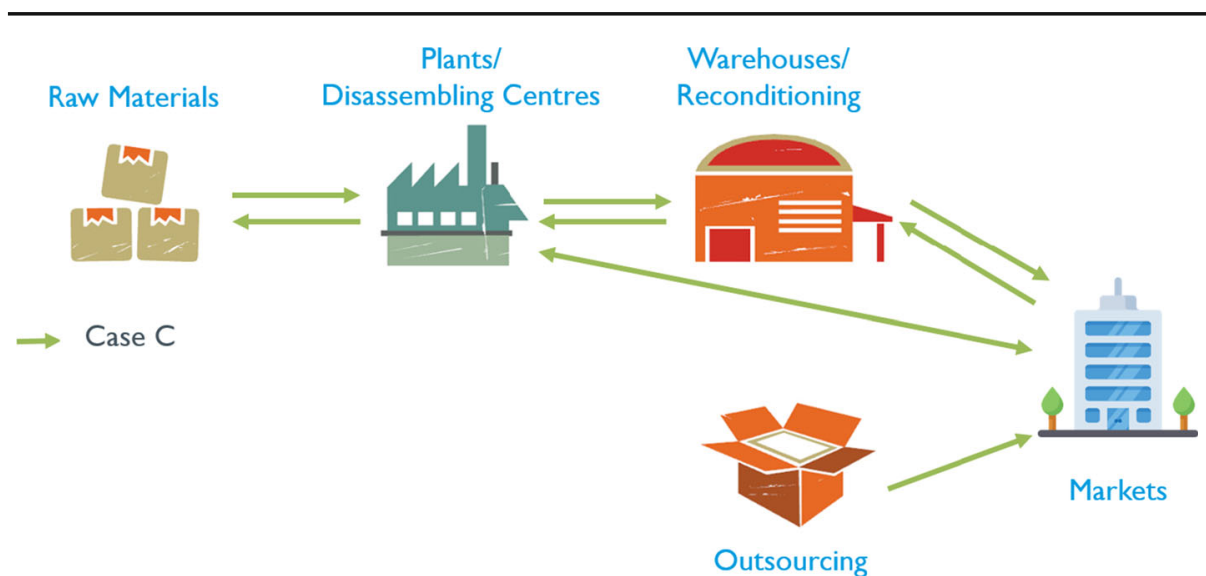

Fig. 8 Schematic configuration of flows allowed in SC Network Configuration C

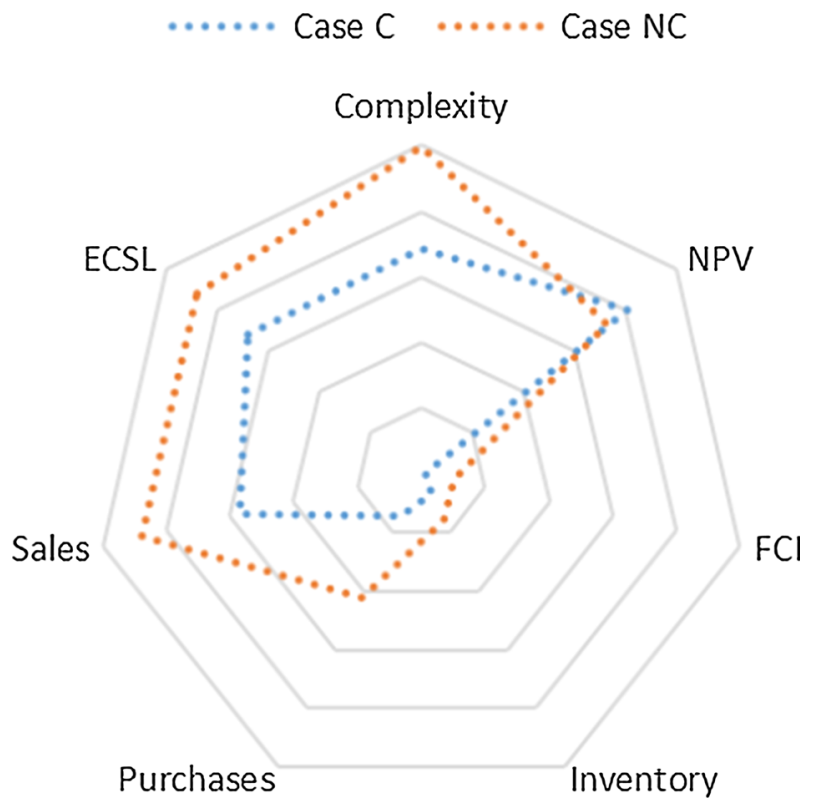

Fig. 9 Average results of SC network configuration $\mathrm{C}$ for the two presented models

3 respectively. Such findings represent a trade-off between increased costs and better ECSL when deploying an operation achieved by the presented model, Fig. 7.

In Case NB, the values for the OF are always higher than those from Case NA, implying a smaller deviation from the ENPV and an improvement in ECSL, when compared to the reference case. This is justified by the higher flexibility, translated into the complexity metric, that characterises this SC configuration.

Supply chain network configuration C Case $\mathrm{C}$ introduces a different kind of flexibility to the network as it allows the same flows as Case B and allows for plants and markets to exchange products without any warehouse intervention, Fig. 8. 


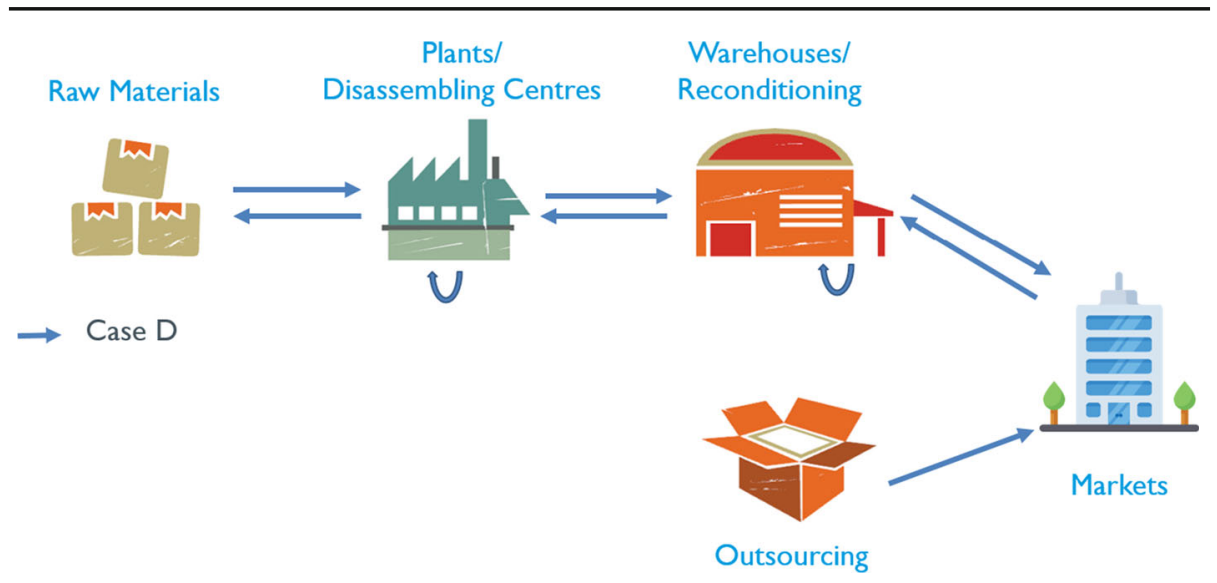

Fig. 10 Schematic configuration of flows allowed in SC Network Configuration D

In this configuration, the results become somehow distinct to Cases A and B. For instance, the results are different from previous runs in the sense that there is not the same trend, being Disruption 2 the one that causes a bigger impact, instead of Disruption 1. However, this configuration still provides better results than SC configurations obtained in Cases A and B. The increased susceptibility to Disruption 2 in Case C, is related with the lower inventory, when compared with the alternative configurations (a characteristic visible in Fig. 9), which in combination with the non-existence of transhipment between entities turn the consequences of failure in supply inevitable.

In Case NC, there is an interesting result when no disruption occurs, the new OF reaches a value above one, which means that for a small decrease in ENPV, the responsiveness of the SC can be improved to levels that almost reach the plenitude of meeting customer demand.

The new metric caused a higher impact on ENPV, with divergences of $-1 \%,-28 \%$ and $2 \%$ for Disruptions 1, 2 and 3 respectively. This means that, while for Disruption 2 the ENPV decreased significantly, for Disruption 3 this new OF provided a network that guarantees a better economic return.

ECSL is benefited in the new SC network configuration as this provides higher levels of service, with variations of $13 \%, 22 \%$ and $2 \%$ for Disruptions 1,2 and 3 respectively. With this, Disruption 2 is not only the event that causes the most economic damage but also the one that provides the higher percentual increase in service quality. The improvement in ECSL is much due to the increase in investment but also due to the increment in purchase activity from outsourcing to meet the demand. The different outcomes for the two models are depicted in Fig. 9.

Supply chain network configuration D Case D allows forward and reverse flows between consecutive echelons and adds a new set of possibilities by allowing transhipment at plants, disassembling centres and warehouses, Fig. 10.

For the economic return driven model, the results are similar to Cases A and B, where Disruption 1 is again, the event that causes the most stress to the operation and its objectives. Such disruption causes a significant decrease in both ENPV and ECSL.

Results for Case ND, when no disruption occurs, show a small decrease in ENPV with an improvement in ECSL, the increase in service quality means to near-total demand fulfilment.

Concerning the maximisation of the SCR performance, Disruption 2 returns the lowest value. This result is due to the high decrease of ENPV, representing a negative variation of 


\section{Case D}

Case ND

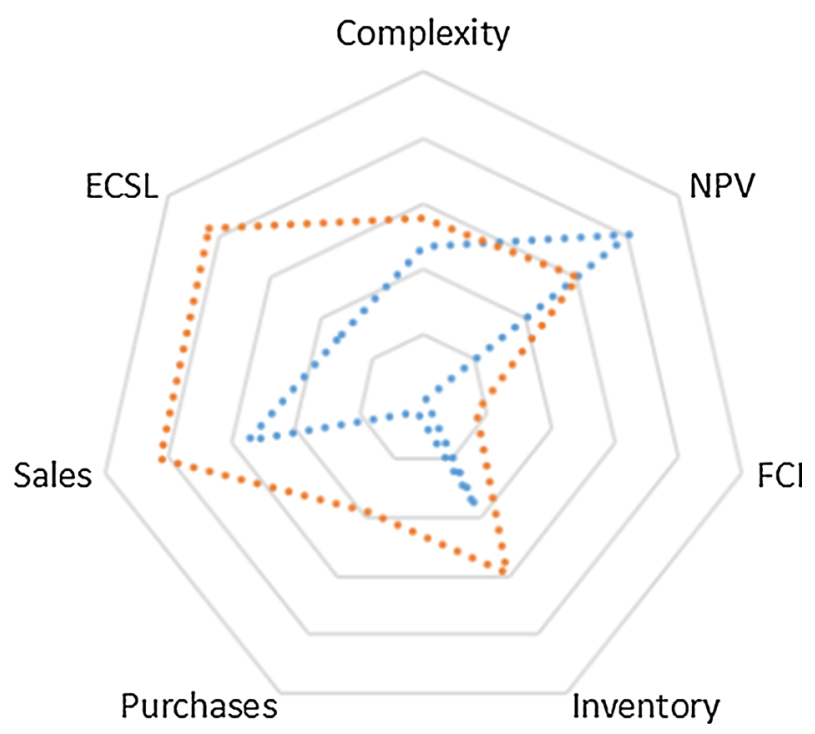

Fig. 11 Average results of SC network configuration D for the two presented models

nearly $50 \%$, which is not compensated by the increase in ECSL. The other disruptions also lead to lower economic returns, however, with smaller proportions representing $-14 \%$, $47 \%$ and $-9 \%$ for Disruptions 1, 2 and 3 respectively.

In line with other cases, due to the introduction of concerns regarding service quality in the OF, ECSL is higher in this model with customer demand being met nearly $90 \%$ of the times of all the disruptions represented, at the expense of increased flow complexity and outsourcing purchases, Fig. 9. This represents an improvement from the reference case of $32 \%, 27 \%$ and $28 \%$ for Disruptions 1, 2 and 3 respectively (11).

Supply chain network configuration E The last case in our study represents a network that includes all the possibilities from previous cases. This closed-loop SC can perform transhipment at all levels, except for markets, and flows bypassing intermediate entities are also allowed, Fig. 12.

From the results, when maximising economic return, it is perceptible that Disruption 1 is the event that influences ENPV the most. Whereas Disruption 2 is the scenario that affects ECSL with an increased impact, with the SC lacking responsiveness and not being able to cope with the lack of supply of raw materials, due to the lower inventory, as shown in Case C (Sect. 6.3.1).

For this case, when no disruption is present, the value of the new resilience metric is higher than one, which means a SC network very similar to that present in the reference case, with the added benefit of the new OF providing the maximum value for ECSL.

While Disruptions 1 and 3 provide a limited impact on ENPV, $-2 \%$ and $3 \%$ respectively, Disruption 2 causes a variation of $-69 \%$ if compared with the reference case. Disruption 2 is the event that originates the lowest resilience metric, caused by the significantly lower 


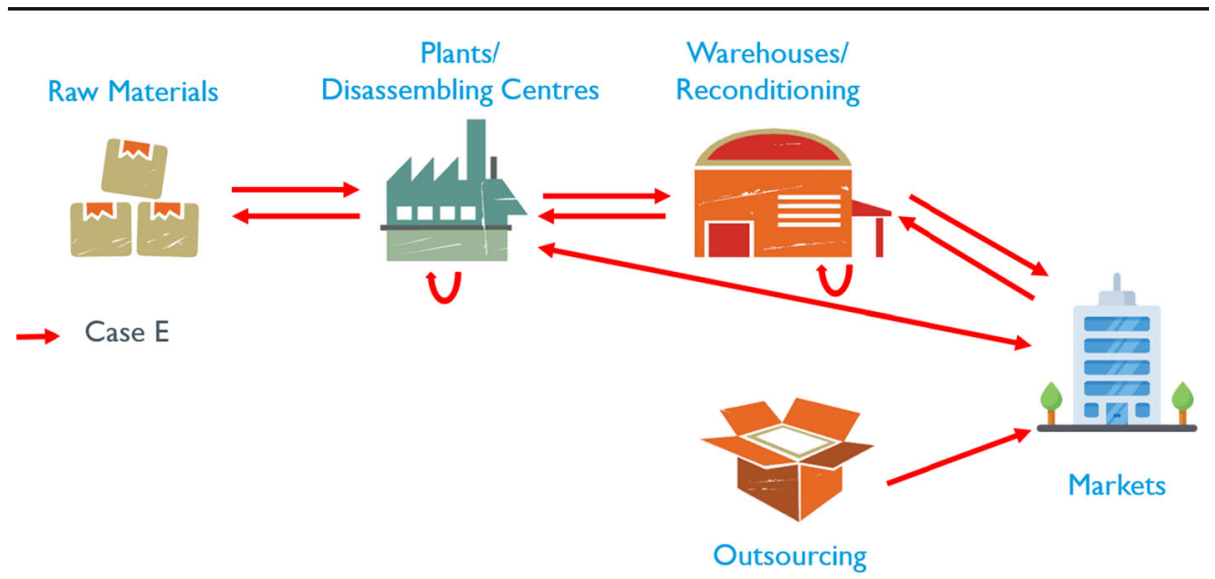

Fig. 12 Schematic configuration of flows allowed in SC Network Configuration E

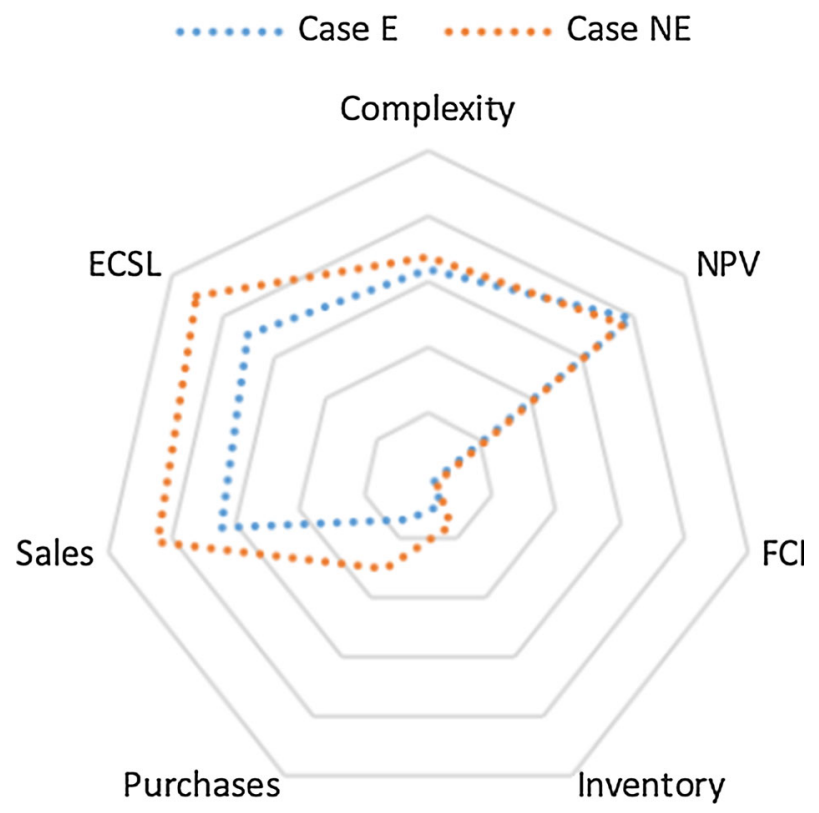

Fig. 13 Average results of SC network configuration E for the two presented models

ENPV, due to the reduction on inventory available. On the contrary, Disruption 3 is able to provide a solution that improves the result from the reference case, although only slightly.

Although the new SC network in case NE provides better ECSL, with all disruptive events reaching values above $92 \%$, it is the case where the percentual variation between the new and reference model results is smaller, Fig. 13, with $12 \%, 19 \%$ and $2 \%$ for Disruptions 1, 2 and 3 respectively. This is much due to the fact of the reference case already reaching better results in this disturbance than in other cases, leaving less space for percentual increases, even if with a good absolute outcome. 


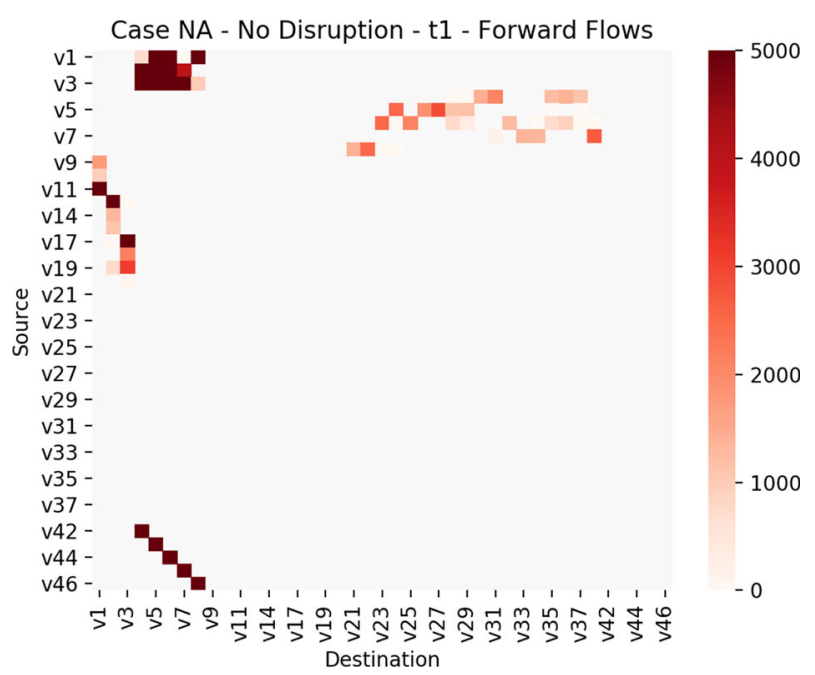

Fig. 14 Forward flows (ton) in Case NA in period 1 when no disruption occurs

\subsubsection{Ripple Effect}

A deeper analysis of the ripple effect is here performed, following the concerns described in the introduction, Sect. 1, regarding the cascading effect on SC caused by disruptions. In the case study considered, four different SC tiers are considered, and the disruptive scenarios were built to represent failures in the different levels, therefore providing the basis for an analysis on how a disruption in one layer can impact other entities of the SC.

For all the different SC network configurations in this case study, there are four distinct types of flows. Take Fig. 14 as an illustrative example:

- Top left corner Flows from factories (v1-v3) to warehouses (v4-v8)

- Middle left Flows from raw materials suppliers (v9-v20) to factories (v1-v3)

- Top right corner Flows from warehouses (v4-v8) to markets (v21-v38)

- Bottom left corner Flows from outsourcing (v43-v50) to warehouses (v4-v8)

Outsourcing entities v46-v50 are implemented in warehouses, v4-v8, and represent an intermediate product that warehouses must buy to sell some products, for example, extra packaging. v43-v45 represent the outsourcing entities of final products ready to be sold.

Using this representation for the flows between different entities in the SC is possible to compare the different disruptive scenarios and infer on the real impact of disruptions and the ripple effect it causes.

Supply chain network configuration A When no disruption occurs, the flows obtained by deploying the new Resilience Metric are presented in Fig. 14. Is visible a high dependency on factory v3 and outsourcing, represented by high colour intensity.

In disruption 1, when one factory (v3) ceases activity in period 2, the effort to fulfil the demand is distributed throughout the remaining working factories, as it can be seen in Fig. 15 , top right corner where the line of $\mathrm{v} 3$ disappeared, and the other lines increased in colour intensity, meaning increased flows intensity. Disruption 3 causes a similar ripple effect when the transportation between a factory (v3) and some warehouses (v4, v6 and v7) cease. The result is a higher workload for the remaining factories, particularly in v1, which can fulfil 


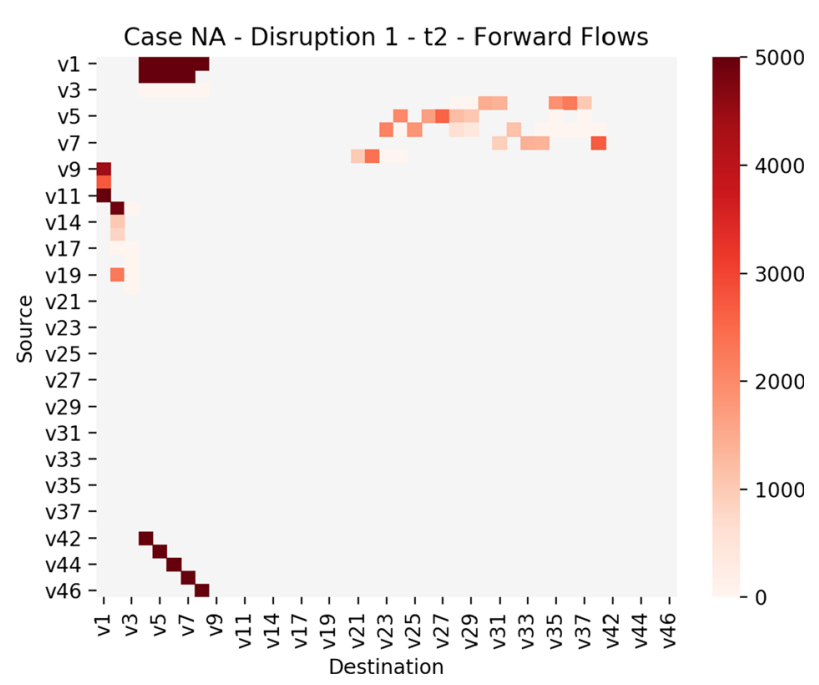

Fig. 15 Forward flows (ton) in Case NA in period 2 for disruption 1

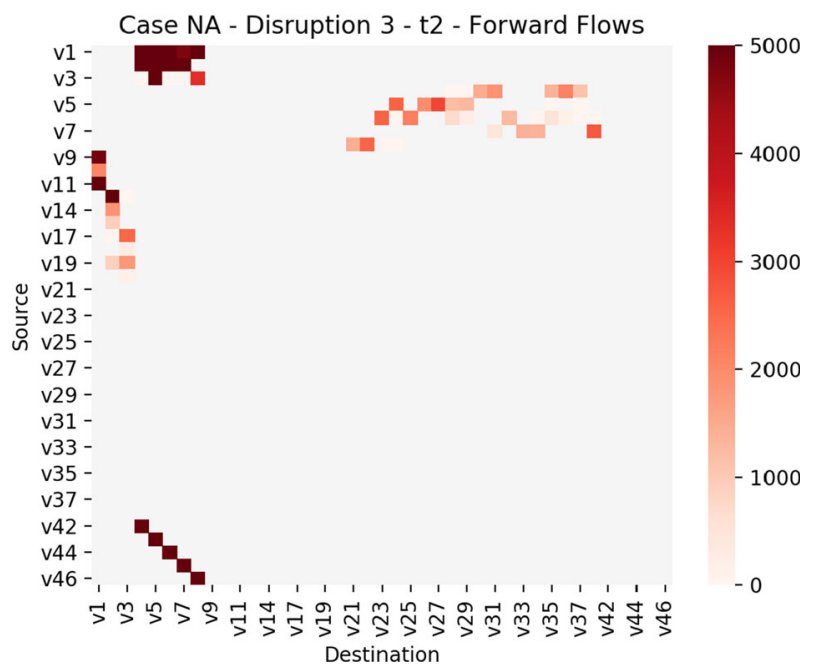

Fig. 16 Forward flows (ton) in Case NA in period 2 for disruption 3

the demand from the affected warehouses, seen by the increased colour intensity for v1 and v2 while most of the flows from v3 disappear in the top left corner in Fig. 16. Consequently, the shifts in the pattern supply/demand lead to higher activity for the flows connecting the raw materials suppliers and the factory (Flow v9 to v1), as shown in Fig. 16.

The above mentioned adverse effects of the ripple effect are diminished using the new resilience metric. If the consequences of the disruptions are compared with the SC network coming from the conventional maximisation of ENPV, then the fluctuation along the SC are much higher, and the negative perception of disruptions for the clients is much higher. Comparing Figs. 17 and 18 with 15 and 16, the beforehand mentioned consequences are visible, with lower quantities reaching to the markets (top right corner), represented by the 


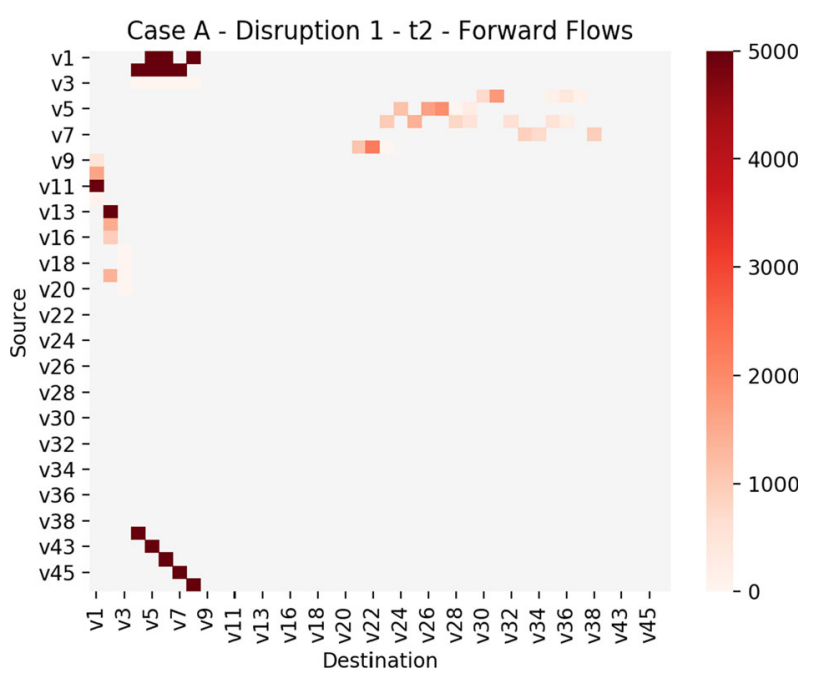

Fig. 17 Forward flows (ton) in Case A in period 2 for disruption 1

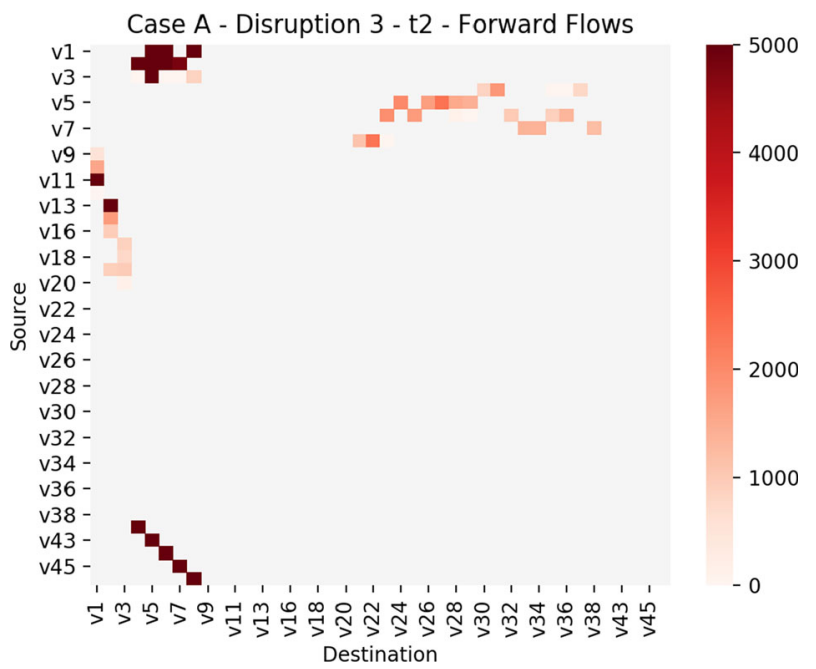

Fig. 18 Forward flows (ton) in Case A in period 2 for disruption 3

faded colour intensity in the flows referring to case A and with higher colour intensity in Case NA, a genuinely detrimental impact of the ripple effect, mitigated by the new resilience metric.

Upstream disruptions, as implemented in Disruption 2, cause the biggest impact, as seen in Sect. 6.3.1, and in terms of the ripple effect along the SC. Not having the suppliers available (v11, v13 and v17 to v19) leads to a reduction in the productive capacity of the SC, worsen by the nonexistence of reverse flows, leading to a reduction in the final flows from warehouses to markets. This inference is visible by comparing the Top Right Corner of Figs. 15, 19 and 16 where the colour intensity is lower when considering Disruption 2. 


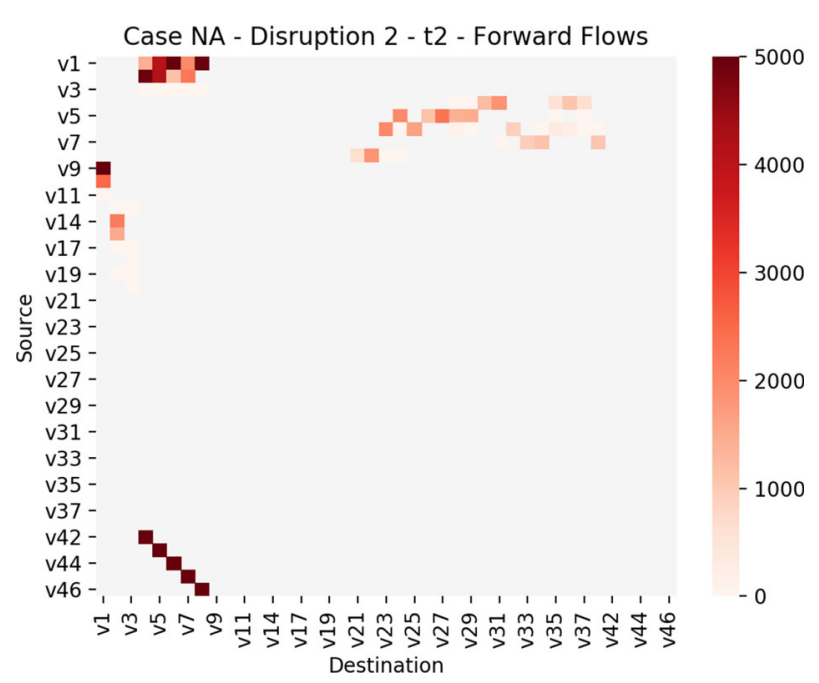

Fig. 19 Forward flows (ton) in Case NA in period 2 for disruption 2

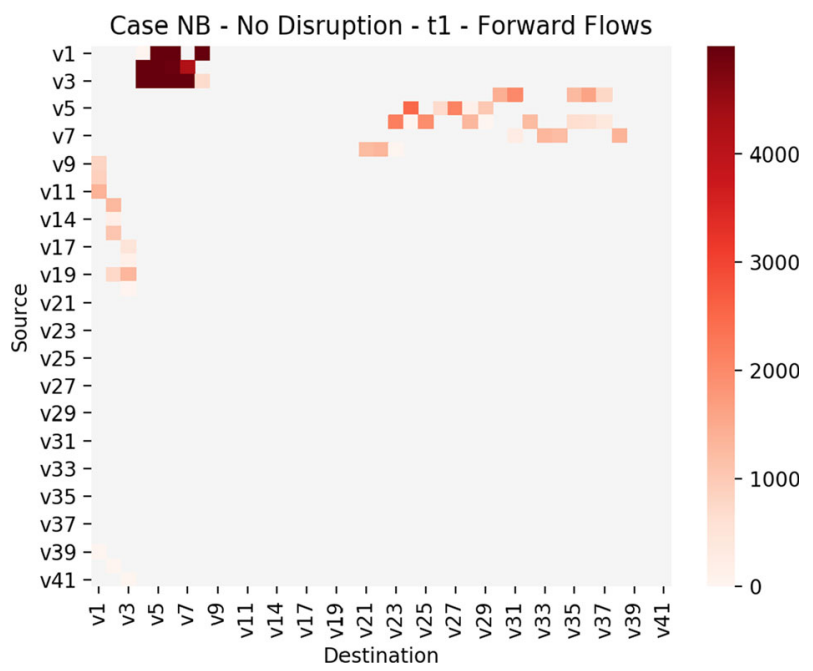

Fig. 20 Forward flows (ton) in Case NB in period 1 when no disruption occurs

Remaining supply chain network configurations As seen in Sect. 6.3.1, so as to maximise NPV and the satisfaction of demand, the SC makes use of reverse flows, discarding the use of outsourcing, see the absence of flows in the bottom left corner of Fig. 20. That is much related to the recovered material coming from reverse flows that are used for the production of products to deliver to markets, thus adding a substantial sustainable benefit.

From Fig. 21 it is possible to see that there is a significant flow of products in the reverse flow, on the left, coming from the markets to the warehouses, a characteristic shared by the different SC network configurations allowing reverse flows. That flow is immediately followed by those in the top right corner representing the flows from the warehouses to 


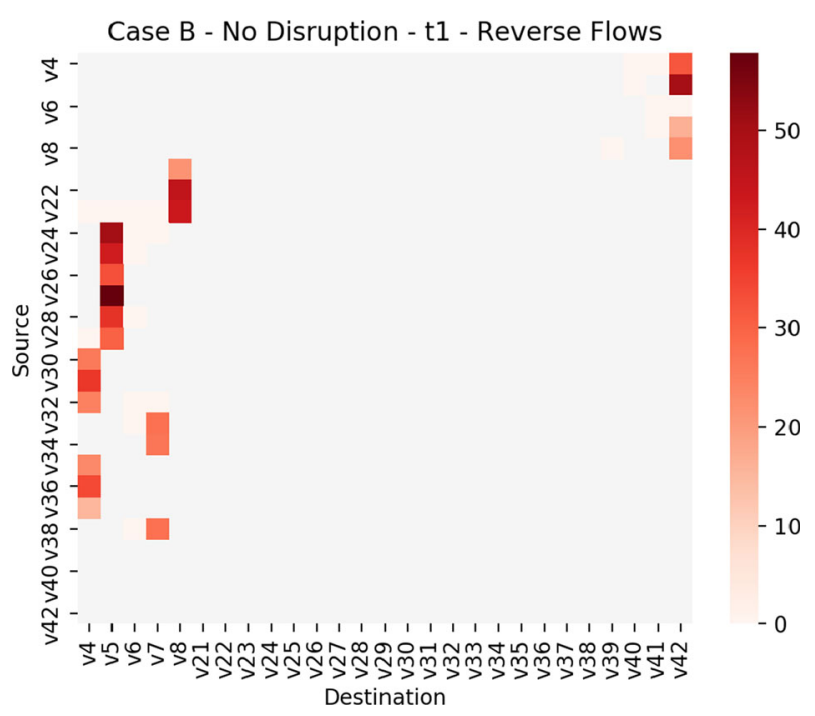

Fig. 21 Reverse flows (ton) in Case B in period 1 when no disruption occurs

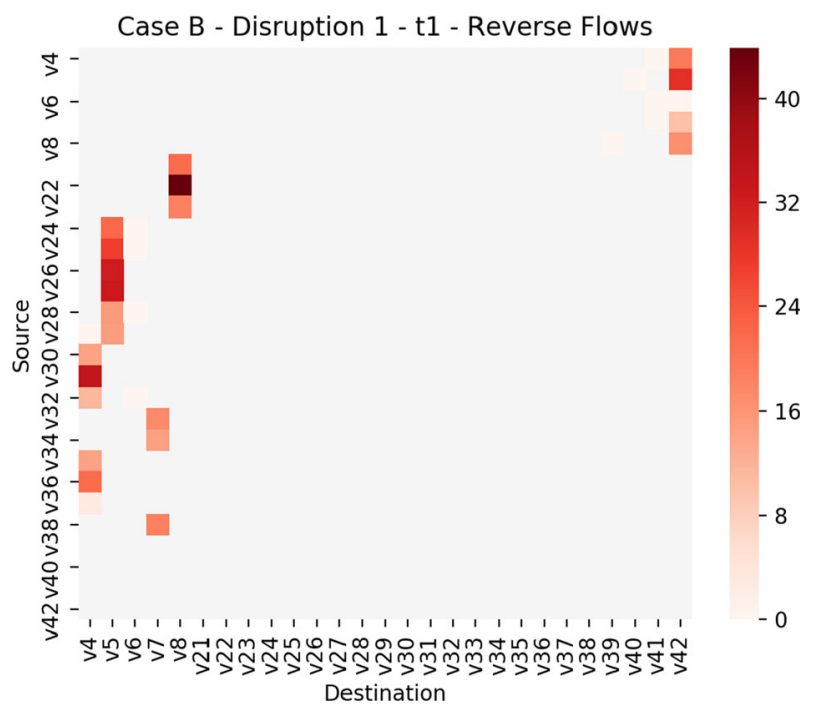

Fig. 22 Reverse flows (ton) in Case B in period 1 when disruption 1 occurs

the facilities which have reverse logistics activities, allowing to be introduced again in the forward SC.

The benefits of the newly introduced resilience metric are quite visible if ENPV maximisation (Figs. 22 and 23) and the New metric (Figs. 24 and 25) are compared when applied the simplest case of a closed-loop SC, in our case study represented by Case B. The reduction in the ripple effect is evident in this example, with a reduction in the variability between consequent time periods. While when maximising ENPV there is a reduction of flows when the description occurs, perceptible in the decrease in colour intensity between Figs. 22 and 23. 


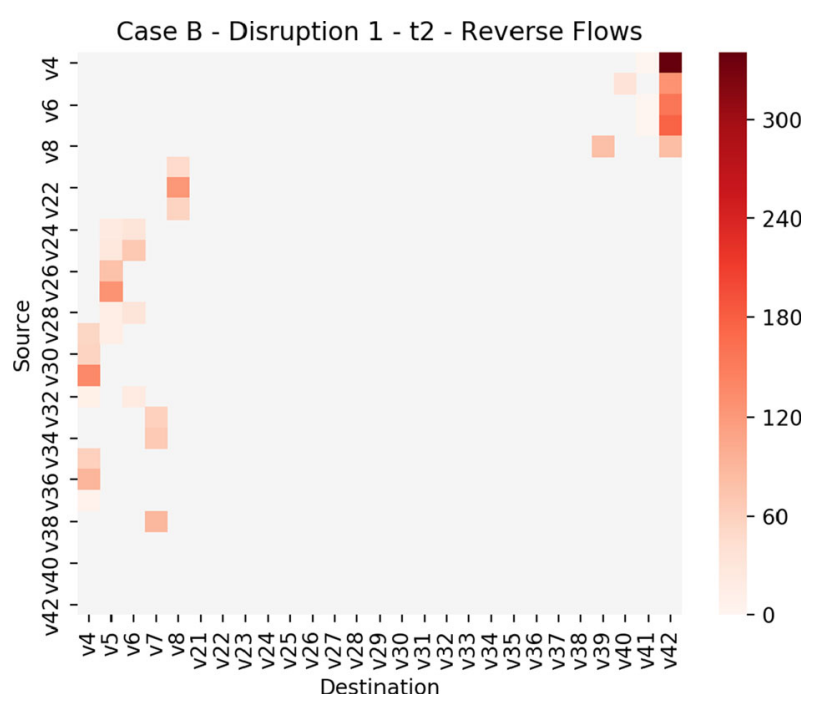

Fig. 23 Reverse flows (ton) in Case B in period 2 when disruption 1 occurs

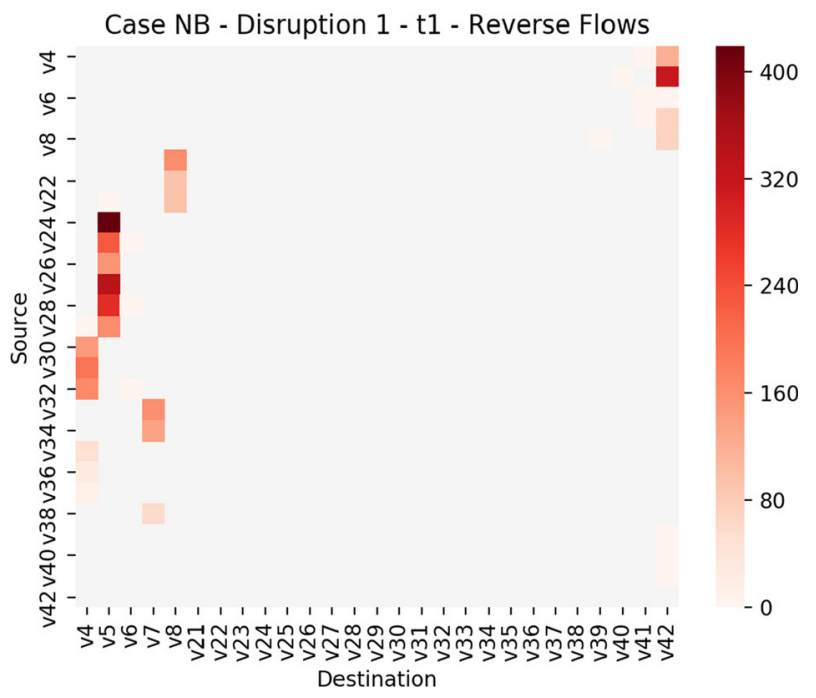

Fig. 24 Reverse flows (ton) in Case NB in period 1 when disruption 1 occurs

The cascading effect is almost imperceptible with the new SCR metric, there is practically no difference in colour intensity between Figs. 24 and 25, thus contributing to a more resilient SC.

When facing a supply disruption, Disruption 2, our results show that the new resilience metric provides a SC better capable of reducing the negative impacts on the consumer, evident when comparing the top right corner of Figs. 26 and 27.

It is relevant to note the different results from SC network configuration $\mathrm{C}$ and $\mathrm{D}$, where the difference between the two cases is the possibility for plants and markets to connect directly for configuration $\mathrm{C}$, and transhipment for configuration D. This added flexibility 


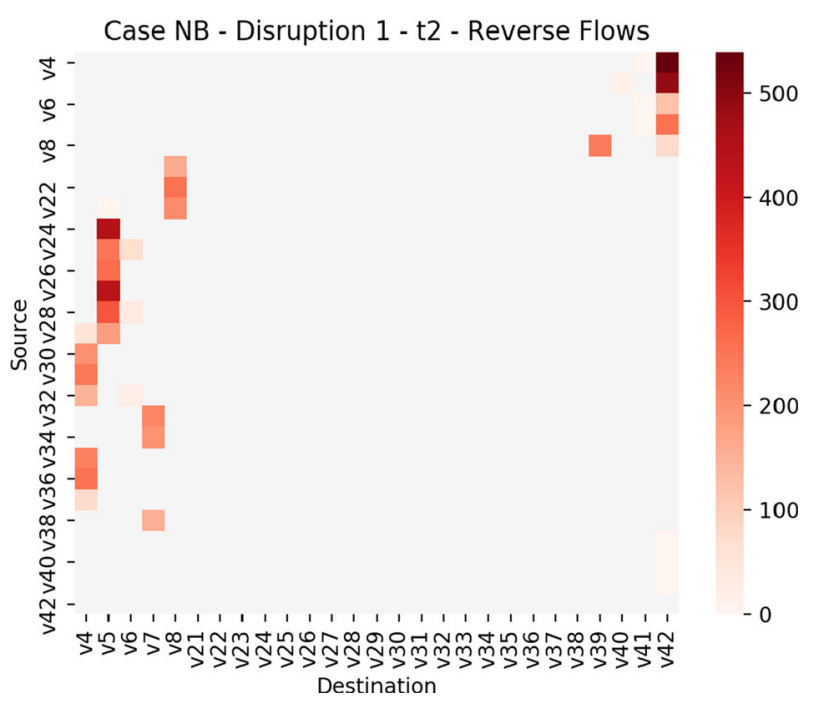

Fig. 25 Reverse flows (ton) in Case NB in period 2 when disruption 1 occurs

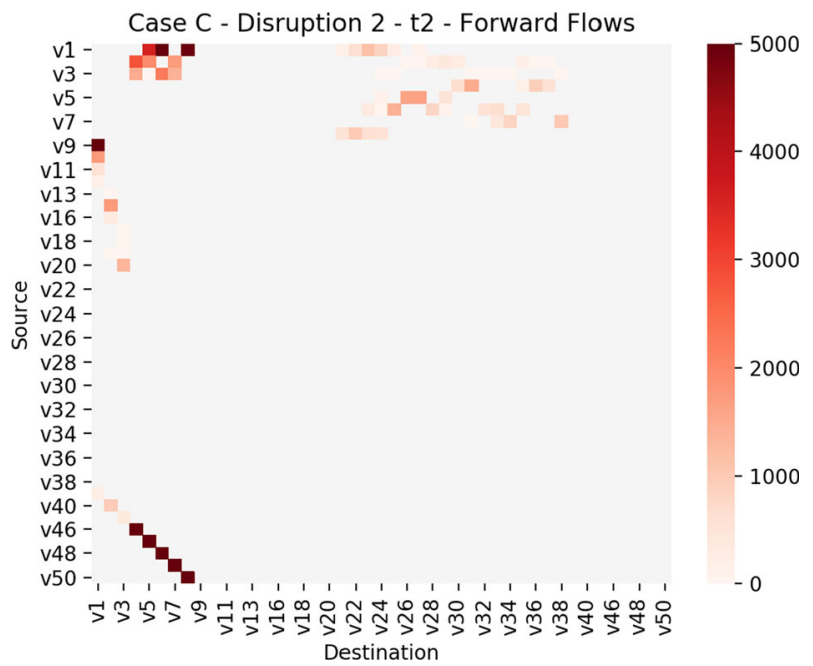

Fig. 26 Forward flows (ton) in Case $\mathrm{C}$ in period 2 when disruption 2 occurs

reduces the severity of the consequences, making use of such possibility to quickly address the disruption. On the other hand, in Case D (Fig. 28) and Case ND (Fig. 29) transhipment does not benefit the response, with an increased negative variation when meeting demand and an increased effort to factories and raw materials suppliers.

The behaviour expressed for network configurations C and D goes well in line with the literature, as discussed in Sect. 1. and further explored in Sects. 7 and 8. 


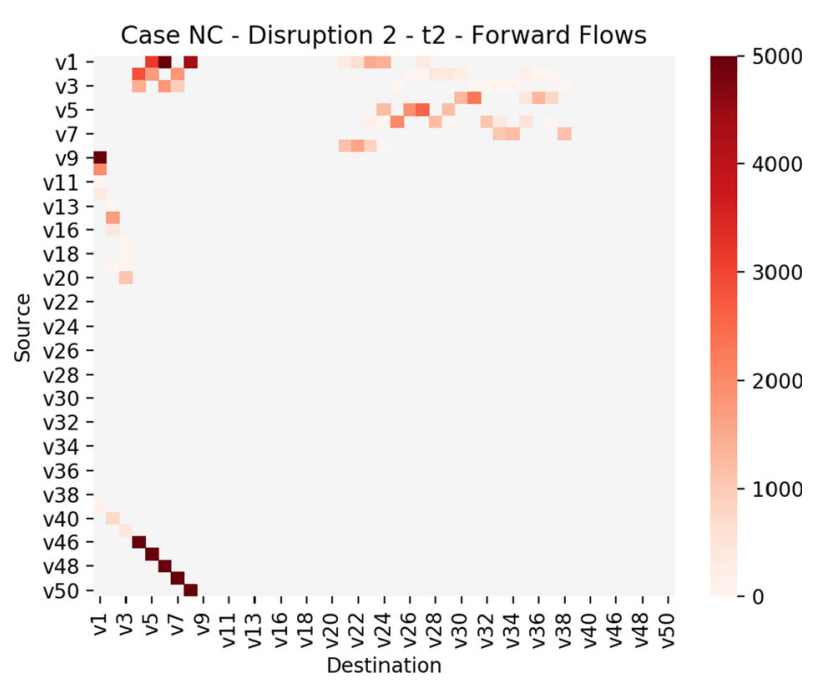

Fig. 27 Forward flows (ton) in Case NC in period 2 when disruption 2 occurs

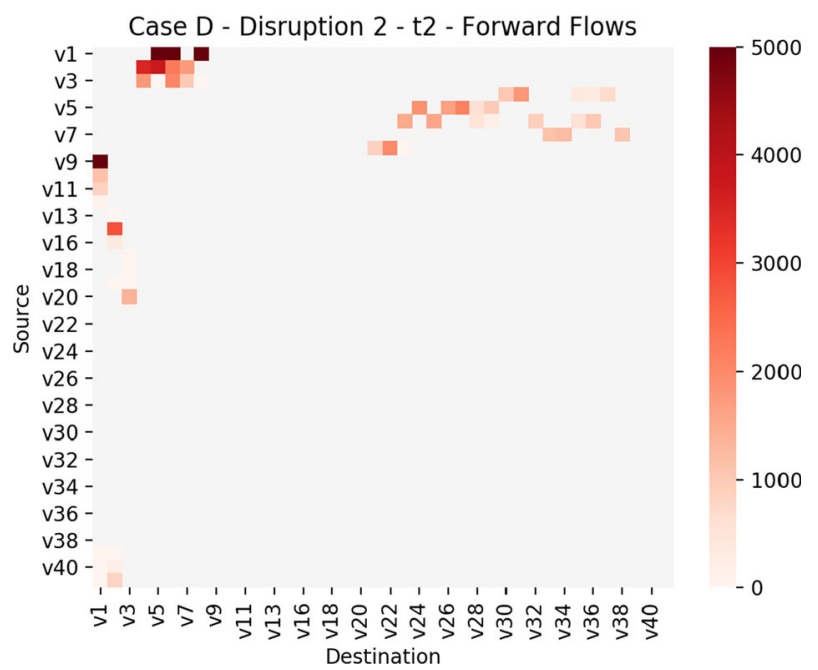

Fig. 28 Forward flows (ton) in Case D in period 2 when disruption 2 occurs

\section{Discussion}

The new resilience metric, translated into the new OF defined in Eq. 12, meets the challenge of generating a quantitative model that better encapsulates the strategic decisions to be made, regarding SC network resilience, in an uncertain environment. Within this approach, the responsiveness of the SC is considered and consequently improved.

Considering that, during the decision-making process, it should be pursued a trade-off between the gains in ECSL and losses in ENPV, a compromise solution between the increases in ECSL and losses in ENPV is targeted. The balance achieved in this balanced metric ensures a better fit between the model results and the challenges created by real scenarios of decision. 


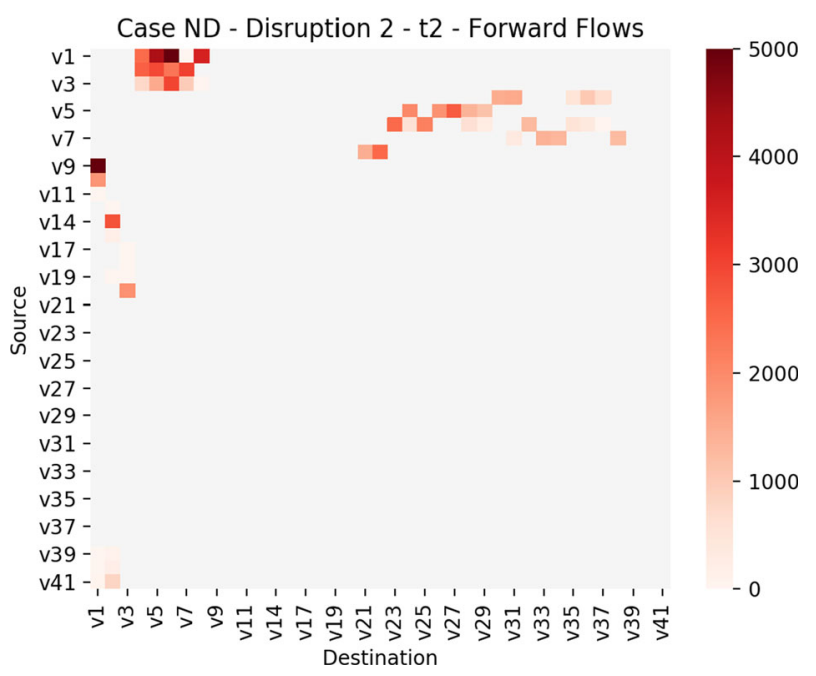

Fig. 29 Forward flows (ton) in Case ND in period 2 when disruption 2 occurs

Failure in this process would lead to solutions that might not be accep for companies, due to low economic results (as the case of maximising complexity) or low service quality (when the main goal is to maximise profit).

The results show that a balance between the different goals, achieved by applying the new metric, is beneficial to companies. The increase in flow redundancy created flexibility, resulting in a proactive strategy to mitigate the costs of disruptions, to keep the operation running while guaranteeing customer's satisfaction.

The case study analysis allows to conclude that it is possible to improve, normal and under disruption operations, with an acceptable amount of investment. The new SC network is responsible for an increase in the SC responsiveness and consequently in the quality of service provided to customers. Additionally, it guarantees an improved company image and consequently, higher success in the markets. That overall benefit is evident in Figs. 5, 7, 9, 11 and 13 where it is possible to see that the SC from the new metric is much better than that obtained by the classical ENPV maximisation.

The increased responsiveness is aligned with the increased ability of the new SCR metric to reduce variability when disruptions occur as well as the ripple effect within the SC. The results presented in Sect. 6.3.2 show that shifting from the current and most commonly used metrics to the new metric results in a reduced cascading effect while maintaining a higher level of customer service.

This is summarised for all cases in Table 4, where the main improvement is seen in ECSL, through the increment in SC responsiveness in all conditions explored, with or without disruptive events.

It is also evident that Disruption 2, a lack of supply, causes the most significant impact on ENPV, for all the Cases considered. This disruption is, however, compensated, based on the new proposed objective, by incrementing the purchase of final products from outsourcing to meet the gap caused by the disruptive event.

Disruption 3 is the event that causes the least amount of variation regarding ENPV, it is even possible, in two cases, to combine the increase of economic return with a better performance in service quality. Thus, the new metric provides a SC that can better cope with disruption 


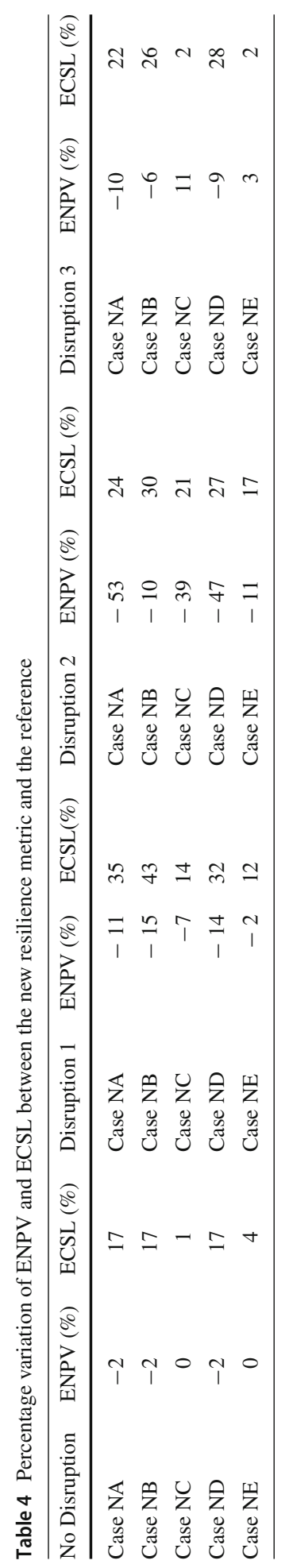


than the SC network from the NPV maximisation. A possible result because the SC network configuration is defined in the steady-state and our metric provides a more balanced network design in the context of SCR, in particular, better prepared for a transportation disruption.

Another critical point is the variation in demand, which was here considered through a scenario approach, see Section 3. This has a noticeable impact on SC performance and consequently, on results. It is the major cause of the decrease in sales, which ultimately causes a reduction in ENPV. The effect of such variation varies from disruption to disruption. For Disruptions 1 and 2, the relative impacts are similar, Table 5.

Disruption 3, a transportation disruption, has a lower variation in ENPV (9\%). In line with what has been said previously, Disruptions 1 and 2 have a variation around $12 \%$ between the worst and best scenarios.

Additionally, from the mean value of the new Resilience Metric for the different runs, Table 6, it is possible to infer that the SC with the lower resilience value is also the simpler SC network, Case NA-Fig. 4. Case ND closely follows, Fig. 10, in the pursuit of improving SC responsiveness, reaches higher levels of ECSL with a more significant reduction of ENPV, which penalises the overall OF value. Case NB provides a network that leads to a substantial increase in ECSL, often with notable drops in economic return. Case NC allows direct flows between plants and markets, adding flexibility to the network by increasing flow complexity since the direct links to markets add redundancy, thus increasing ECSL. However, the increased flexibility is not able to cope with Disruption 2, an event that disrupts the supply side of the SC and where the SC cannot take advantage of the multitude of flows. To meet customer demand, the SC has to rely on outsourcing, reaching outstanding levels of service quality in an evident trade-off with the economic return. This shows that this management alternative should also be considered when planning SC. The case with fewer limitations, Case NE, although providing a SC network similar to Case NC, does not return the SC with the higher resilient behaviour, since the increased investment leads to lower financial results when facing some types of disruptive events.

\subsection{Comparison with other SCR Metrics}

To the best of our knowledge, there are four publications that provide a single index metric for the resilience of a SC. It is relevant to compare how similar those are with the metric here presented and the pros and cons of each.

Hosseini and Ivanov (2019) provides a Bayesian approach to a resilience metric applied to the context of SC the ratio between recovery and loss presented by Henry and Emmanuel Ramirez-Marquez (2012). This metric is devoted to an operational level of SCM, where time has to be precisely described. This characteristic unable its use for strategic decisions, naturally with a multitude of periods. Due to its different objectives, any comparison between the results from the two metrics is rendered unpractical.

Azevedo, Carvalho, and Cruz-Machado (2016) proposed an index obtained from four vectors (Lean, Agile, Resilient and Green) that ultimately provide a metric for resilience. However, this was done with the automotive industry in mind, and the adaptation to a generic $\mathrm{SC}$ is not trivial, in fact can hardly be done. The model is quantified in a scale from 1 to 5 in terms of implementation of resilient practices or the perceived ability of the company for a particular behaviour. This approach is highly dependent on the collection of data from the SC and the company operation since it relies on the identification of practices and/or educated guesses on the SC capability, which, as mentioned before in this paper, can be an extremely 


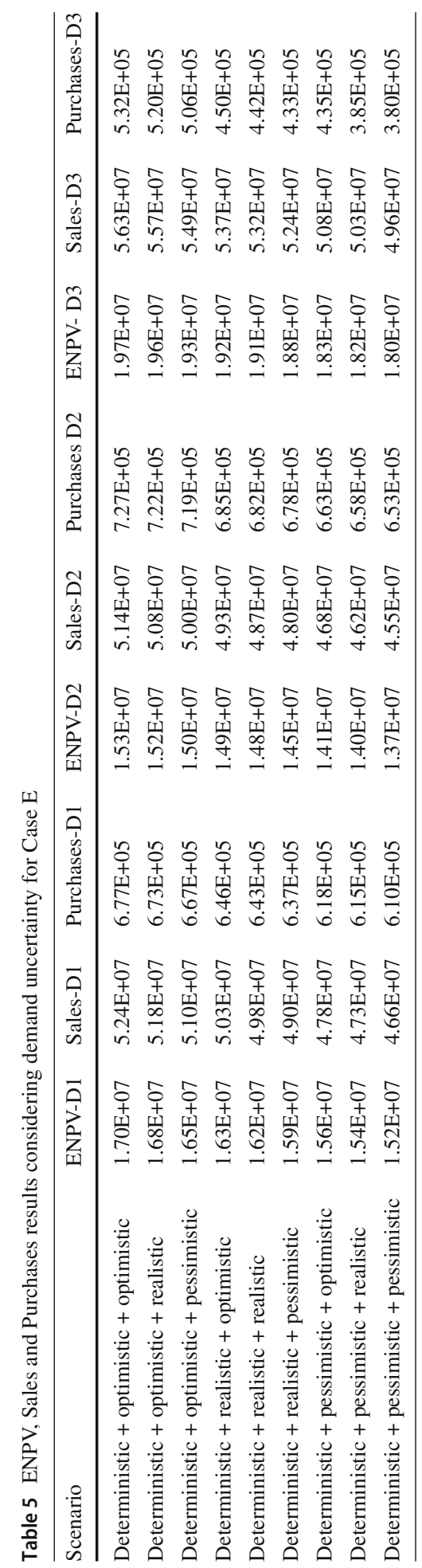


Table 6 Mean resilience metric values for cases NA to NE
Mean resilience metric value

0.665

0.811

0.749

0.695

0.705

difficult task in the context of SCR. As we aim to have a generic metric, the quantitative comparison with this metric renders difficult, as its focus is mainly operational.

Soni, Jain, and Kumar (2014)'s approach to measuring SCR relies on the identification of resilience enablers, recurring to a survey with practitioners and academics. The enablers found are in line with the literature (information, collaboration, agility, sustainability, risk and revenue sharing, trust, visibility, risk management culture, adaptive capability and structure) and, as in the previous work, fall under more operational objectives that entail qualitative interpretation of the SC operation.

Wang and Ip (2009)'s resilience metric is focused on the aircraft industry and is a far simpler approach to the problem. It takes into account the supply and demand needed for a continuous flow of aeroplanes. Nevertheless, this simplification reduces the depth of the analysis. The case study applied considers a two-tier SC, reducing the replicability of the metric dramatically to different case studies or industries, turning it impractical to compare with other SC.

In sum, the published metrics are often applied to operational problems, leaving untouched the strategic level of decision. In fact, it was impossible to use any of the published SCR metrics since those are restrictive in terms of the industry for which they were designed. Moreover, the metrics are operational or reliant on qualitative considerations that disable the possibilities of comparison between SC, when considering quantitative decision support approaches. Thus, there is a research gap identified which has been tackled in this paper. A new resilience metric is created from the combination of the two factors (economic and responsiveness). This resilience metric allows for a comparison between several possible cases and to infer on what are the more resilient cases. A positive correlation between what is the immediate interpretation of results with the value of the resilience metric was observed, where SC with poor results have a correspondent low level in the newly proposed metric.

\section{Managerial insights}

There is a need to acknowledge that SCR capabilities are essential to prepare for unforeseen events. And in this context, the deployment of decision-making aiding tools is crucial for a well-designed resilient SC. Our results show that a specific, balanced metric is beneficial for companies to improve their resilient behaviour, improving its SC responsiveness while reducing the ripple effect.

Decision-makers should then use OR methods that aim for a balanced solution network, which will inform on a more resilient operation more capable of dealing with a multitude of events. Focusing on one type of events can be restrictive and leave out vulnerabilities in the SC. Thus, companies should invest in strategies aimed at mitigating diverse supply disruptions, being in this way prepared to reduce the harmful impacts in the long term. 
In this set, increasing flow complexity is considered a good strategy to be adopted. However, it must be done in a trade-off with the related increasing costs. In our results, with the new proposed SCR metric, a balanced solution is obtained that improves the responsiveness, reducing the ripple effect while maintaining good operational results. Combining economic supply chain goals and customer service levels as objectives is then an enabler to a more resilient SC that should be pursued by the SC managers.

In conclusion, the only possible mindset to face disruptive scenarios is by proactively guarantee customer needs. The ultimate value for a SC is to provide goods to be sold and failing to do so, the main consequence of a SC disruption, can endanger the companies involved. The pursuit for a continuous selling activity is crucial, even if running the danger of lowering financial results in steady-state operations. The proposed metric helps to reach this goal.

\section{Conclusions and future work}

In this paper, a SCR quantitative model is presented, addressing the research gap of SCR metrics and the applicability of OR methods to the field of SC under resilience concerns.

We start by characterising a model to study SCR considering a set of resilience metrics, among which a new one is proposed. The latter is constructed to allow replicability and transferability between different applications, following the research gap identified.

The new model leads to SC that can better cope with the challenge of meeting customer demand while providing an easy way to measure and compare SCR.

It is imperative to relate the new findings to those already acknowledged in previous publications. While some general conclusions can be drawn, some results come from the particular nature of the combinations between the SC network, the case study, and the different disruptions.

The model developed considers some assumptions, such as the number of levels in the SC (four) and the uncertainty presence just on demand and disruptions. These aspects may be the focus of further research to incorporated more SC characteristics and contexts, leading to a more detailed model in terms of SC structure and uncertainty presence (supply; technologies, disruptions occurrence). Furthermore, the metric developed is dependent on the reference SC data. This may limit a generalised application of the proposed resilience quantification, and so further studies on different SC may be developed so as to acquire more knowledge on the application of the proposed resilience metric.

From the work developed, it can be stated that pro-active resilient strategies can have a positive impact on SC performance, by reducing the ripple effect, increasing its responsiveness, and service quality can be significantly improved. However, the understanding of SCR drivers is still an underdeveloped topic, with a perceived lack of how different drivers shape a SC operation under disruption. There is an evident need for further work on the impact that network complexity causes on SCR, especially to provide an insight into the relationship between increases in SC flow complexity and increased responsiveness and resilience.

The food SC is an interesting case study due to the perishability of products that create a particularity. A stop in sales leads not only to lost sales but also a direct cost in food spoiled, lost inventory. Therefore is could be a economic sector that would benefit from further study on SCR.

SCR is, in conclusion, a topic where there is space for improvement. Our work contributes to the development of SCR by presenting a novel resilience metric that can be applied to any 
SC and provide usable results that can be used to aid decision-making. There is still space for further testing the presented metric on the resilient behaviour, by applying it to different Case Studies and improving the knowledge on the resilience drivers to better understand the resilient performance of SC.

Acknowledgements The authors acknowledge the support provided by FCT - Foundation for Science and Technology, I.P., under the project UIDB/00097/2020 and P2020 under the project PTDC/EGEOGE/28071/ 2017, Lisboa -01.0145-Feder-28071 and SFRH/BD/148499/2019.

\section{Annex 1: model}

Sets

\begin{tabular}{|c|c|}
\hline $\mathrm{V}$ & Set of entities/locations \\
\hline $\mathrm{P}$, & Set of products \\
\hline $\mathrm{S}$, & Set of nodes in the scenario tree \\
\hline $\mathrm{I}$, & Set of available technologies \\
\hline$I_{\text {pro }} \subseteq I$ & Set of processing technologies \\
\hline$I_{d e s} \subseteq I$ & Set of disassembling technologies \\
\hline$P_{f} \subseteq P$ & Set of final products \\
\hline $\operatorname{Prod}_{f} \subseteq P$, & Set of final products from reverse flows \\
\hline$F_{v, w}$ & Set of pairs $(\mathrm{v}, \mathrm{w})$ such that a forward flow is allowed between $\mathrm{v}$ and $\mathrm{w}$, \\
\hline$R_{v, w}$ & Set of pairs $(\mathrm{v}, \mathrm{w})$ such that a reverse flow is allowed between $\mathrm{v}$ and $\mathrm{w}$, \\
\hline $\mathrm{T}$, & Set of time periods \\
\hline$N_{t} \subseteq S$ & Set of scenarios of time period $\mathrm{t} \in \mathrm{T}$ \\
\hline pred $_{r} \subseteq S$ & Set of scenarios that precede scenario $r$ in the scenario tree, \\
\hline predsec $_{r} \subseteq S$ & Set of scenarios that precede in two time periods scenario $r$ in the scenario tree, \\
\hline$V_{w h} \subseteq V$ & Set of entities with warehousing \\
\hline$V_{t r} \subseteq V$ & Set of transforming entities \\
\hline$V_{w r} \subseteq V$ & Set of warehouses with reverse logistics technologies \\
\hline$V_{m} \subseteq V$ & Set of markets \\
\hline$V_{g} \subseteq V$ & Set of suppliers \\
\hline$V_{f} \subseteq V$ & Set of factories \\
\hline$V_{l} \subseteq V$ & Set of warehouses \\
\hline$V_{c} \subseteq V$ & Set of entities with technologies $n$ \\
\hline$V_{e} \subseteq V$ & Set of eliminating entities \\
\hline$V_{d} \subseteq V$ & Set of disassembling entities \\
\hline$O U T_{p} \subseteq I$ & Set of technologies that produce product $\mathrm{p} \in \mathrm{P}$ \\
\hline$O U T j_{p} \subseteq I$ & Set of technologies that produce product $\mathrm{p} \in \mathrm{P}$ from reverse logistics \\
\hline$I N_{p} \subseteq I$ & Set of technologies that consume product $\mathrm{p} \in \mathrm{P}$ \\
\hline$I N J_{p} \subseteq I$ & Set of technologies that produce product $\mathrm{p} \in \mathrm{P}$ from reverse logistics \\
\hline$M P_{i} \subseteq P$ & Set of final products for each technology $i \in I$ \\
\hline$M P J_{i} \subseteq P$ & Set of final products for each technology $i \in I$ from reverse logistics \\
\hline$P U R_{v} \subseteq P$ & Set of products that can be bought by entity $\mathrm{v} \in \mathrm{V}$ \\
\hline
\end{tabular}


Parameters

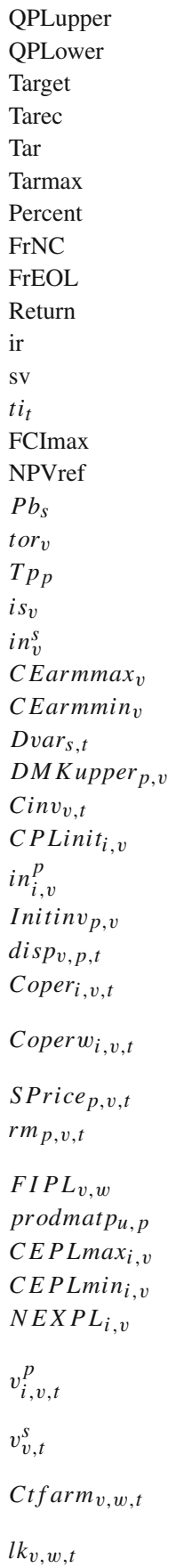

Upper bound for the flow of materials between entities in time period 1

Lower bound for material flow between entities in time period 1

Minimum percentage of demand satisfaction

Minimum percentage of collection of non-compliant products

Minimum percentage of end-of-life product collection in markets

Maximum percentage of end-of-life product collection in markets

Minimum percentage utilisation of each technology's installed capacity

Percentage of non-conforming collected products that are disposed

Percentage of end-of-life products that are disposed of

Percentage of non-conforming products on the markets

Interest rate

Residual value of investment

Tax rate in time period $\mathrm{t} \in \mathrm{T}$

Maximum value for total invested capital

Value for reference NPV

Probability of occurrence of each node $s \in S$

Turnover rate for entity $v \in V$

Service life of final products $\mathrm{p} \in P_{f}$

Initial investment associated with each entity with storage, $\mathrm{v} \in V_{w h}$

Initial capacity of each entity with storage, $v \in V_{w h}$

Maximum value for capacity expansion of entity $\mathrm{v} \in V_{w h}$

Min value for capacity expansion of entity $\mathrm{v} \in V_{w h}$

Demand variation for scenario $s \in S$ for periods $t \in T$ greater than 1

Demand of product $p \in P$ on entity $v \in V$ in scenario 1 in time period 1

Inventory cost of entity $v \in V$ over time $t \in T$ in euros per year

Initial technology capability $i \in I$ in entity $v \in V$

Initial investment associated with each technology $i \in I$ in each entity $v \in V$

Initial inventory of product $p \in P$ on entity $v \in V$

Availability of raw material $p \in P$ at supplier $v \in V$ within time $t \in T$

Operating cost of entity $v \in V$ and technology $i \in I$ per unit produced in euros per ton in time period $t \in T$

Operating cost of entity $v \in V$ and technology $i \in I$ per unit produced in euros per ton in time period $t \in T$

Price of final product sold at entity $v \in V$ over time $t \in T$ in euros per ton

Price of raw material $p \in P$ sold by entity $v \in V$ in time period $t \in T$ in euros per ton

Distance between entities $v, w \in V$

Amount of raw materials $u \in P$ needed for each $p \in P$

Maximum limit for capacity expansion of each entity $v \in V$ and technology $\mathrm{i} \in \mathrm{I}$

Minimum limit for technology $i \in I$ capacity expansion in each entity $v \in V$

Maximum number of capacity expansions allowed for each process $i \in I$ in each entity $v \in V$

Variable investment associated with each technology $i \in I$ in each entity $v \in V$ in each time period $t \in T$ in euros

Variable investment associated with each entity $v \in V$ with storage in time period $t \in T$ in euros

Unit cost of transporting a product from one entity $v \in V$ to another $w \in V$ in time period $t \in T$ in euros per ton.km

Contract cost of a carrier to make a journey from one entity $v \in V$ to another $w \in V$ in the period $t \in T$ in euros 
Decision Variables

$\mathrm{z}$

$P U_{v, w, p, s, t}$

$W_{i n} n_{i, v, p, s, t}$

Winw $_{i, v, p, s, t}$

Wout $_{i, v, p, s, t}$

Wout $w_{i, v, p, s, t}$

$Q_{v, w, p, s, t}$

$Q N C_{v, w, p, s, t}$

$Q E L_{v, w, p, s, t}$

$S A_{v, p, s, t}$

$I N V_{v, p, s, t}$

$I L_{v, s, t}$

$i m_{i, v}$

$C E_{i, v, t}^{p}$

Carm $_{v, t}$

$C E_{v, t}^{s}$

$N P V_{S}$

$C F_{S, t}$

$N E_{S, t}$

$F D C_{t}$

FCI

$D E P_{t}$

$Y_{v, w, t}$

$Y N C_{v, w, t}$

$Y E L_{v, w, t}$

$D_{p, v, s, t}$

Transpt

Product $_{t}$

Purchasest

Inventory

Salest

I $D_{p, v, s, t}$

CServicet

Invest

CF tot $_{t}$

nodes $_{v, t}$

NPVtotal
Objective variable

Purchases of product $p \in P$ made by entity $v \in V$, from entity $w \in V$, in scenario $s \in S$ and in time period $t \in T$

Flow of product $p \in P$ which is consumed by technology $i \in I$ in entity $v \in V$, in time period $t \in T$ and scenario $s \in S$

Product flow $p \in P$ which is consumed by technology $i \in I$ in entity $v \in V$, in time period $t \in T$ and scenario $s \in S$

Flow of the product $p \in P$ which is produced by technology $i \in I$ in entity $v \in V$, in time period $t \in T$ and scenario $s \in S$

Flow of product $p \in P$ which is produced by technology $i \in I$ in entity $v \in V$, in time period $t \in T$ and scenario $s \in S$

Product flow $p \in P$ sent from entity $v \in V$ to entity $w \in V$ in time period $t \in T$ and scenario $s \in S$

Product $p \in P$ reverse flow not as sent from entity $v \in V$ to entity $w \in V$ in time period $t \in T$ and scenario $s \in S$

End-of-life product $p \in P$ reverse flow sent from entity $v \in V$ to entity $w \in V$ in time period $t \in T$ and scenario $s \in S$

Sales value of each product $p \in P$ on market $v \in V$ for time period $t \in T$ and scenario $s \in S$

Inventory of product $p \in P$ kept in entity $v \in V$ in time period $t \in T$ and scenario $s \in S$

Mean inventory level maintained at entity $v \in V$ in each time period $t \in T$ and scenario $s \in S$

Capacity of technology $i \in I$ in entity $v \in V$

Technology $i \in I$ capacity expansion in entity $v \in V$ over time period $t \in T$

Storage capacity of entity $v \in V$ over time period $t \in T$

Storage expansion of entity $v \in V$ over time period $t \in T$

Net present value in scenario $s \in S$

Cash flow in time period $t \in T$ and scenario $s \in S$

Profit after Tax in time period $t \in T$ and scenario $s \in S$

Fraction of total depreciable capital that has to be paid over time period $t \in T$

Fixed Capital Investment

Depreciation term in time period $t \in T$

Binary variable equal to 1 if the forward link between entity $v \in V$ and entity $w \in V$ is established at time period $t \in T$ and 0 otherwise

Binary variable equal to 1 if the reverse link between entity $v \in V$ and entity $w \in V$ is established at time period $t \in T$ and 0 otherwise

Binary variable equal to 1 if the end-of-life reverse link between entity $v \in V$ and entity $w \in V$ is established at time period $t \in T$ and 0 otherwise

Demand for each product $\mathrm{p} \in \mathrm{P}$, in each entity $\mathrm{v} \in \mathrm{V}$, in the scenario $s \in S$ and at time period $\mathrm{t} \in \mathrm{T}$

Cost of transportation in time period $\mathrm{t} \in \mathrm{T}$

Cost of production in time period $t \in T$

Cost in purchases in time period $t \in T$

Cost with inventory in time period $\mathrm{t} \in \mathrm{T}$

Value of sales in time period $\mathrm{t} \in \mathrm{T}$

Unsatisfied demand of product $p \in P$ in entity $v \in V$ for scenario $s \in S$ in time period $\mathrm{t} \in \mathrm{T}$

Customer service in time period $\mathrm{t} \in \mathrm{T}$

Cost with investment in time period $\mathrm{t} \in \mathrm{T}$

Total cash flow in time period $t \in T$

Binary variable equal to 1 if entity $v \in V$ opens in period $t \in T$ and 0 otherwise

NPV considering all scenarios 
Objective function

$\mathrm{z}=\sum_{s \in S}\left(\frac{\mathrm{pb}_{s} \cdot \mathrm{NPV}_{s}}{\mathrm{NPVref}}\right)-\sum_{t \in T}\left(\frac{\text { CService }_{t}}{|T|}\right)$

Constraints

Constraint $1_{v, p, s, t}$

$\sum_{w \in V} \mathrm{PU}_{v, w, p, s, t}+\sum_{i \in \mathrm{OUT}_{i, p}}$ Wout $_{i, v, p, s, t}+\sum_{w \in \mathrm{F}_{w, v}} \mathrm{Q}_{w, v, p, s, t}=\sum_{w \in \mathrm{F}_{v, w}} \mathrm{Q}_{v, w, p, s, t}+$ $\sum_{i \in \mathbb{I N}_{i, p}} \operatorname{Win}_{i, v, p, s, t} \quad \forall v \in V, p \in P, t \in T, s \in \mathrm{N}_{t}$

Constraint $2_{s, t}$

$\sum_{v \in V, w, p \in \mathrm{PUR}_{p, v}} \mathrm{PU}_{v, w, p, s, t} \leq 0 \quad \forall t \in T, s \in \mathrm{N}_{t}$

Constraint $3 v, p, s, t$

$\sum_{w \in V} \mathrm{PU}_{w, v, p, s, t} \leq \operatorname{disp}_{v, p, t} \quad \forall v \in V, p \in P, t \in T, s \in \mathrm{N}_{t}$

Constraint $4_{i, v, u, s, t}$
Win $_{i, v, u, s, t}=\sum_{p \in \mathrm{MP}_{p, i}}\left(\right.$ Wout $\left._{i, v, p, s, t} \cdot \operatorname{prodmatp}_{u, p}\right) \forall i \in I_{p r o}, v \in V_{t r}, u \in P, t \in T, s \in \mathrm{N}_{t}$

\section{Constraint $5 v, p, s, t$}

$\sum_{r \in \operatorname{pred}_{s, r}} \mathrm{INV}_{v, p, r, t-1}+\sum_{w \in V_{f}} \mathrm{Q}_{w, v, p, s, t}+\sum_{w \in V_{l}} \mathrm{Q}_{w, v, p, s, t}+\sum_{w \in V} \mathrm{PU}_{v, w, p, s, t}$

$+\sum_{i \in \mathrm{OUT}_{i, p}}$ Wout $_{i, v, p, s, t}+\sum_{w \in \mathrm{R}_{w, v}} \mathrm{QNC}_{w, v, p, s, t}+\sum_{w \in \mathrm{R}_{w, v}} \mathrm{QEL}_{w, v, p, s, t}=\sum_{w \in V_{m} w} \mathrm{Q}_{v, w, p, s, t}+$

$\sum_{w \in V_{l}} \mathrm{Q}_{v, w, p, s, t}+\mathrm{INV}_{v, p, s, t}+\sum_{i \in \mathrm{IN}_{i, p}} \mathrm{Win}_{i, v, p, s, t}+\sum_{x \in V_{e}} \mathrm{QNC}_{v, x, p, s, t}+\sum_{x \in V_{e}} \mathrm{QEL}_{v, x, p, s, t}+$

$\sum_{x \in V_{d}} \mathrm{QEL}_{v, x, p, s, t} \quad \forall v \in V_{l}, p \in P, t \in(T \wedge t>1), s \in \mathrm{N}_{t}$

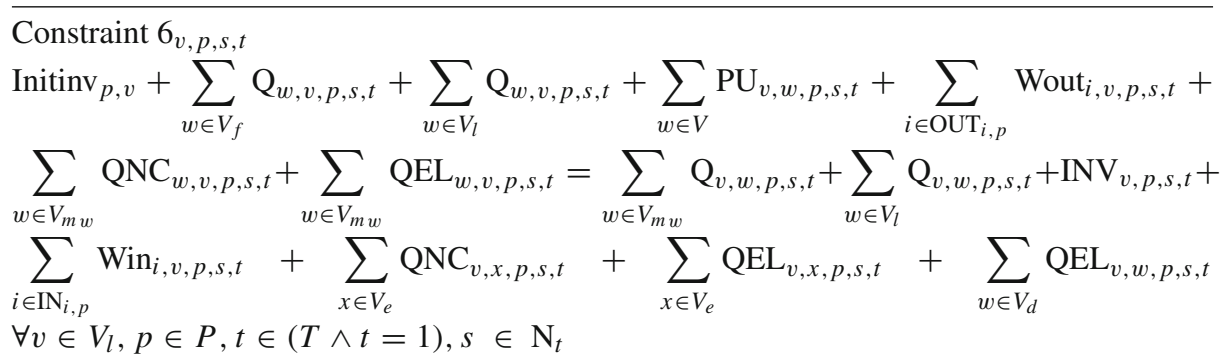

Constraint $7 v, p, s, t$

$\mathrm{SA}_{v, p, s, t}=\sum_{w \in \mathrm{F}_{w, v}} \mathrm{Q}_{w, v, p, s, t} \quad \forall v \in V_{m}, p \in P, t \in T, s \in \mathrm{N}_{t}$

Constraint $8_{v, p, s, t}$

$\mathrm{SA}_{v, p, s, t} \geq \mathrm{D}_{p, v, s, t} \cdot \operatorname{target} \quad \forall v \in V_{m}, p \in P, t \in T, s \in \mathrm{N}_{t}$ 
Constraint $9_{v, p, s, t}$

$\mathrm{SA}_{v, p, s, t} \leq \mathrm{D}_{p, v, s, t}$

$\forall v \in V_{m}, p \in P, t \in T, s \in \mathrm{N}_{t}$

Constraint $10_{i, v, s, t}$

$\sum_{p \in \mathrm{MP}_{p, i}}$ Wout $_{i, v, p, s, t} \leq \operatorname{im}_{i, v, t} \quad \forall i \in \mathrm{I}_{\text {pro }}, v \in V_{t r}, t \in T, s \in \mathrm{N}_{t}$ )

Constraint $11_{i, v, s, t}$

Percent $\cdot \operatorname{im}_{i, v, t} \leq \sum_{p \in \mathrm{MP}_{p, i}}$ Wout $_{i, v, p, s, t} \quad \forall i \in \mathrm{I}_{p r o}, v \in V_{t r}, t \in T, s \in \mathrm{N}_{t}$ )

Constraint $12_{i, v, s, t}$

$\operatorname{im}_{i, v, t} \geq \sum_{p \in \mathbb{I N}_{i, p}} \operatorname{Win}_{i, v, p, s, t} \quad \forall i \in \mathrm{I}_{p r o}, v \in V_{t r}, t \in T, s \in \mathrm{N}_{t}$ )

Constraint $13_{i, v, t}$

$\operatorname{im}_{i, v, t}=\operatorname{im}_{i, v, t-1}+\mathrm{CE}_{i, v, t} \quad \forall i \in I, v \in V_{c}, t \in(T \wedge t>1)$

Constraint $14_{i, v, t}$

$\operatorname{im}_{i, v, t}=$ CPLinit $_{i, v}+\mathrm{CE}_{i, v, t} \quad \forall i \in I, v \in V_{c}, t \in(T \wedge t=1)$

Constraint $15_{i, v, t}$

$\mathrm{CE}_{i, v, t} \leq \mathrm{CEPLmax}_{i, v}$

$\forall i \in I, v \in V_{c}, t \in T$

Constraint $16_{i, v, t}$

$\mathrm{CE}_{i, v, t} \geq \mathrm{CEPLmin}_{i, v}$

$\forall i \in I, v \in V_{c}, t \in T$

Constraint $17_{i, v}$

$\sum_{t \in T} \mathrm{CE}_{i, v, t} \leq \mathrm{NEXPL}_{i, v}$

$\forall i \in I, v \in V_{c}$

Constraint $18_{v, s, t}$

$\sum_{p \in P} \operatorname{INV}_{v, p, s, t} \leq \operatorname{Carm}_{v, t}$

$\forall v \in V, t \in T, s \in \mathrm{N}_{t}$

Constraint $19_{v, s, t}$

$2 \cdot \mathrm{IL}_{v, s, t} \leq \mathrm{Carm}_{v, t}$

$\forall v \in V, t \in T, s \in \mathrm{N}_{t}$

Constraint $20_{v, s, t}$

$\mathrm{IL}_{v, s, t}=\frac{\sum_{w \in F_{v, w}, p \in P} \mathrm{Q}_{v, w, p, s, t}}{\text { tor }_{v}} \quad \forall v \in V, t \in T, s \in \mathrm{N}_{t}$

Constraint $21_{v, t}$

$\operatorname{Carm}_{v, t}=\operatorname{Carm}_{v, t-1}+\mathrm{CE}_{v, t}^{s}$

$\forall v \in V, t \in(t>1)$

Constraint $22_{v, t}$

$\operatorname{Carm}_{v, t}=\mathrm{in}_{v}^{s}+\mathrm{CE}_{v, t}^{s}$

$\forall v \in V, t \in(t=1)$

Constraint $23_{v, t}$

$\mathrm{CE}^{s}{ }_{v, t} \leq \mathrm{CEarmmax}_{v}$

$\forall v \in V_{z}, t \in T$ 
Constraint $24_{v, t}$

$\mathrm{CE}_{v, t}^{s} \geq \mathrm{CEarmmin}_{v}$

$\forall v \in V_{z}, t \in T$

Constraint $25_{v}$

$\sum_{t \in T} \mathrm{CE}_{v, t}^{s} \leq \mathrm{NEXarm}_{v}$

Constraint $26_{v, w, s, t}$

$\sum_{p \in P}\left(\mathrm{Q}_{v, w, p, s, t}+\mathrm{PU}_{v, w, p, s, t}\right) \leq \mathrm{QPLupper} \cdot \mathrm{Y}_{v, w, t} \quad \forall(v, w) \in \mathrm{F}_{v, w}, t \in T, s \in \mathrm{N}_{t}$

Constraint $27_{v, w, s, t}$

$\sum_{p \in P}\left(\mathrm{Q}_{v, w, p, s, t}+\mathrm{PU}_{v, w, p, s, t}\right) \geq$ QPLower $\cdot \mathrm{Y}_{v, w, t} \quad \forall(v, w) \in \mathrm{F}_{v, w}, t \in T, s \in \mathrm{N}_{t}$

Constraint $28_{s}$

$\mathrm{NPV}_{s}=\sum_{t \in \mathrm{N}_{t}}\left(\frac{\mathrm{CF}_{s, t}}{(1+\mathrm{ir})^{t}}\right)$

Constraint $29_{s, t}$

$\mathrm{CF}_{s, t}=\mathrm{NE}_{s, t}-\mathrm{FDC}_{t}$

$\forall t \in\left(T \wedge t \leq(|T|-1), s \in \mathrm{N}_{t}\right.$

Constraint $30_{s, t}$

$\mathrm{CF}_{s, t}=\mathrm{NE}_{s, t}-\mathrm{FDC}_{t}-\mathrm{sv} \cdot \mathrm{FCI} \quad \forall t \in\left(T \wedge t=|T|, s \in \mathrm{N}_{t}\right.$

Constraint $31_{s, t}$

$\mathrm{NE}_{s, t}=(1-\mathrm{ti}) \cdot\left(\sum_{v \in V_{m}, p \in P}\left(\right.\right.$ SPrice $\left._{p, v, t} \cdot \mathrm{SA}_{v, p, s, t}\right)-\sum_{v \in V_{m}, w \in V, p \in P,(v, w) \in R}\left(\mathrm{QNC}_{v, w, p, s, t}\right.$.

SPrice $\left._{p, v, t}\right)-\sum_{v \in V, w \in V_{g}, p \in P}\left(\mathrm{rm}_{p, w, t} \cdot \mathrm{PU}_{v, w, p, s, t}\right)-\sum_{i \in I, v \in V_{t r}, p \in P}\left(\operatorname{Coper}_{i, v, t} \cdot\right.$ Wout $\left._{i, v, p, s, t}\right)-$

$\sum_{i \in I, v \in V_{d}, p \in P}\left(\operatorname{Coperw}_{i, v, t} \cdot\right.$ Woutw $\left._{i, v, p, s, t}\right)-\sum_{v \in \mathrm{V}}\left(\operatorname{Cinv}_{v, t} \cdot \mathrm{IL}_{v, s, t}\right)-\sum_{v \in V, w \in V, p \in P}\left(\operatorname{Ctfarm}_{v, w, t} \cdot\right.$ $\left.\left.\left(\mathrm{Q}_{v, w, p, s, t}+\mathrm{QNC}_{v, w, p, s, t}+\mathrm{QEL}_{v, w, p, s, t}+\mathrm{PU}_{w, v, p, s, t}\right) \cdot \mathrm{FIPL}_{v, w}\right)\right)+\mathrm{ti} \cdot \mathrm{DEP}_{t} \forall t \in T, s \in \mathrm{N}_{t}$

Constraint $32_{t}$

$\mathrm{DEP}_{t}=\frac{(1-\mathrm{sv}) \cdot \mathrm{FCI}}{|T|} \quad \forall t \in T$

Constraint 33

$\mathrm{FCI}=\sum_{i \in I, v \in V_{c}, t \in T}\left(\mathrm{v}^{p}{ }_{i, v, t} \cdot \mathrm{CE}_{i, v, t}\right)+\sum_{i \in I, v \in V}\left(\mathrm{im}_{i, v} \cdot \mathrm{in}_{i, v}\right)+\sum_{v \in \mathrm{V}}\left(\mathrm{in}_{v}^{s} \cdot \mathrm{is}_{v}\right)+\sum_{v, t \in \mathrm{V}}\left(\mathrm{v}_{v, t}^{s} \cdot\right.$

$\left.\mathrm{CE}_{v, t}^{s}\right)+\sum_{v \in V, w \in V, t \in T}\left(\mathrm{lk}_{v, w, t} \cdot\left(\mathrm{Y}_{v, w, t}+\mathrm{YNC}_{v, w, t}+\mathrm{YEL}_{v, w, t}\right)\right)$

Constraint 34

FCI $\leq$ FCImax

Constraint $35_{t}$

$\mathrm{FDC}_{t}=\frac{\mathrm{FCI}}{|t|}$ 
Constraint $36_{v, p, s, t}$
$\sum_{x \in \mathrm{R}} \mathrm{QEL}_{v, x, p, s, t} \geq$
$\sum_{\left(\mathrm{F}_{w, v} \wedge \operatorname{pred}_{s, r}\right)}$
$\mathrm{Q}_{w, v, p, r, t-\mathrm{Tp}_{p}} \cdot \operatorname{tar}\left[\left(\mathrm{Tp}_{p} \geq 2\right)\right]$
$\mathrm{Q}_{w, v, p, r, t-\mathrm{Tp}_{p}} \cdot \operatorname{tar}\left[\left(\mathrm{Tp}_{p} \leq 1\right)\right]+$
$\sum$
$w, r \in\left(\mathrm{F}_{w, v} \wedge \operatorname{predsec}_{s, r}\right)$ $\forall v \in V_{m}, p \in \operatorname{Prod}_{f}, t \in T, s \in \mathrm{N}_{t}$

Constraint $37_{v, p, s, t}$

$\sum_{x \in \mathrm{R}} \mathrm{QEL}_{v, x, p, s, t} \leq \sum_{w, r \in\left(\mathrm{F}_{w, v} \wedge \operatorname{pred}_{s, r}\right)} \mathrm{Q}_{w, v, p, r, t-\mathrm{Tp}_{p}} \cdot \operatorname{tarmax}\left[\left(\mathrm{Tp}_{p} \leq 1\right)\right]+\sum_{w, r \in\left(\mathrm{F}_{w, v} \wedge \operatorname{predsec}_{s, r}\right)}$

$\mathrm{Q}_{w, v, p, r, t-\mathrm{Tp}_{p}} \cdot \operatorname{tarmax}\left[\left(\mathrm{Tp}_{p} \geq 2\right)\right] \quad \forall v \in V_{m}, p \in \operatorname{Prod}_{f}, t \in T, s \in \mathrm{N}_{t}$

Constraint $38_{v, p, s, t}$

$\sum_{w \in V_{e}} \mathrm{QNC}_{v, w, p, s, t}=\mathrm{FrNC} \cdot \sum_{x \in V_{m_{x}}} \mathrm{QNC}_{x, v, p, s, t} \quad \forall v \in V_{l}, p \in P, t \in T, s \in \mathrm{N}_{t}$

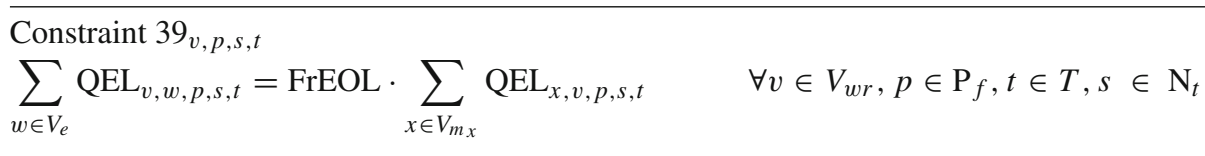

Constraint $40_{v, p, s, t}$

$\sum_{w \in \mathrm{R}_{v, w}} \mathrm{QNC}_{v, w, p, s, t} \geq \sum_{w \in \mathrm{F}_{w, v}} \mathrm{Q}_{w, v, p, s, t} \cdot$ return $\cdot \operatorname{tarec} \quad \forall v \in V_{m}, p \in P, t \in T, s \in \mathrm{N}_{t}$

Constraint $41_{v, p, s, t}$

$\sum_{w \in \mathrm{R}_{v, w}} \mathrm{QNC}_{v, w, p, s, t} \leq \sum_{w \in \mathrm{F}_{w, v}} \mathrm{Q}_{w, v, p, s, t} \cdot$ return $\quad \forall v \in V_{m}, p \in P, t \in T, s \in \mathrm{N}_{t}$

Constraint $42_{i, v, u, s, t}$

Winw $_{i, v, u, s, t}=\sum_{p \in \mathrm{MPJ}_{p, i}}\left(\right.$ Woutw $\left._{i, v, p, s, t} \cdot \operatorname{prodmatp}_{u, p}\right) \forall i \in \mathrm{I}_{d e s}, v \in V_{d}, u \in P_{f}, t \in T, s$ $\in \mathrm{N}_{t}$

Constraint $43_{v, p, s, t}$

$\sum_{i \in \mathrm{OUTj}_{i, p}}$ Woutw $_{i, v, p, s, t}+\sum_{w \in \mathrm{R}_{w, v}} \mathrm{QEL}_{w, v, p, s, t}=\sum_{w \in \mathrm{F}_{v, w}} \mathrm{Q}_{v, w, p, s, t}+\sum_{i \in \mathrm{INJ}_{i, p}} \mathrm{Winw}_{i, v, p, s, t}+$ $\sum_{w \in V_{e}} \mathrm{QEL}_{v, w, p, s, t}$

$\forall v \in V_{d}, p \in P, t \in T, s \in \mathrm{N}_{t}$

Constraint $44_{i, v, s, t}$

$\sum_{p \in \mathrm{MPJ}_{p, i}}$ Woutw $_{i, v, p, s, t} \leq \operatorname{im}_{i, v, t} \quad \forall i \in \mathrm{I}_{d e s}, v \in V_{d}, t \in T, s \in \mathrm{N}_{t}$

Constraint $45_{i, v, s, t}$

Percent $\cdot \operatorname{im}_{i, v, t} \leq \sum_{p \in \mathrm{MPJ}_{p, i}}$ Woutw $_{i, v, p, s, t} \quad \forall i \in \mathrm{I}_{d e s}, v \in V_{d}, t \in T, s \in \mathrm{N}_{t}$

Constraint $50_{v, p, s, t}$

$\sum_{w \in \mathrm{R}_{w, v}} \mathrm{QEL}_{w, v, p, s, t}-\sum_{w \in V_{e}} \mathrm{QEL}_{v, w, p, s, t}=\sum_{i \in \mathrm{INJ}_{i, p}} \mathrm{Winw}_{i, v, p, s, t} \forall v \in V_{d}, p \in P, t \in T, s$ $\in \mathrm{N}_{t}$ 
Constraint $51_{v, w, s, t}$

$\sum_{p \in P} \mathrm{QNC}_{v, w, p, s, t} \leq$ QPLupper $\cdot \mathrm{YNC}_{v, w, t} \quad \forall(v, w) \in \mathrm{R}_{v, w}, t \in T, s \in \mathrm{N}_{t}$

Constraint $52_{v, w, s, t}$

$\sum_{p \in P} \mathrm{QNC}_{v, w, p, s, t} \geq$ QPLower $\cdot \mathrm{YNC}_{v, w, t} \quad \forall(v, w) \in \mathrm{R}_{v, w}, t \in T, s \in \mathrm{N}_{t}$

Constraint $53_{v, w, s, t}$

$\sum_{p \in \operatorname{prod}_{f}} \mathrm{QEL}_{v, w, p, s, t} \leq$ QPLupper $\cdot \mathrm{YEL}_{v, w, t} \quad \forall(v, w) \in \mathrm{R}_{v, w}, t \in T, s \in \mathrm{N}_{t}$

Constraint $54_{v, w, s, t}$

$\sum \mathrm{QEL}_{v, w, p, s, t} \geq \mathrm{QPLower} \mathrm{YEL}_{v, w, t} \quad \forall(v, w) \in \mathrm{R}_{v, w}, t \in T, s \in \mathrm{N}_{t}$ $p \in \operatorname{prod}_{f}$

Constraint $55_{v, p, s, t}$

$\sum_{w \in V_{m} w} \mathrm{QEL}_{w, v, p, s, t}-\sum_{w \in V_{e}} \mathrm{QEL}_{v, w, p, s, t}=\sum_{w \in V_{d}} \mathrm{QEL}_{v, w, p, s, t} \forall v \in V_{l}, p \in P, t \in T, s \in \mathrm{N}_{t}$

Constraint $56_{i, v, s, t}$

$\operatorname{im}_{i, v, t} \geq \sum_{p \in \mathrm{INJ}_{i, p}}$ Winw $_{i, v, p, s, t} \quad \forall i \in \mathrm{I}_{d e s}, v \in V_{d}, t \in T, s \in \mathrm{N}_{t}$

Constraint $57_{v, p, s, t}$

$\left.\mathrm{D}_{p, v, s, t}=\sum_{r \in \operatorname{pred}_{s, r}} \mathrm{D}_{p, v, r, t-1} \cdot \operatorname{Dvar}_{s, t}\right) \quad \forall v \in V_{m}, p \in \mathrm{P}_{f}, t \in(T \wedge t>1), s \in \mathrm{N}_{t}$

Constraint $58_{v, p, s, t}$

$\mathrm{D}_{p, v, s, t}=$ DMKupper $_{p, v} \quad \forall v \in V_{m}, p \in \mathrm{P}_{f}, t \in(T \wedge t=1), s \in \mathrm{N}_{t}$

Constraint $59_{t}$

$\operatorname{Transp}_{t}=\sum_{s \in S, v \in V, w \in V, p \in P}\left(\mathrm{pb}_{s} \cdot\left(\mathrm{Ctfarm}_{v, w, t} \cdot\left(\mathrm{Q}_{v, w, p, s, t}+\mathrm{QNC}_{v, w, p, s, t}+\mathrm{QEL}_{v, w, p, s, t}+\right.\right.\right.$

$\left.\left.\left.\mathrm{PU}_{w, v, p, s, t}\right) \cdot \mathrm{FIPL}_{v, w}\right)\right)+\sum_{v \in V, w \in V}\left(\mathrm{lk}_{v, w, t} \cdot\left(\mathrm{Y}_{v, w, t}+\mathrm{YNC}_{v, w, t}+\mathrm{YEL}_{v, w, t}\right)\right) \quad \forall t \in T$

Constraint $60_{t}$

Produc $_{t}=\sum_{s \in S, i \in I, v \in \text { Trans }_{v}, p \in P}\left(\mathrm{pb}_{s} \cdot\left(\right.\right.$ Coper $_{i, v, t} \cdot$ Wout $\left.\left._{i, v, p, s, t}\right)\right) \quad \forall t \in T$

Constraint $61_{t}$

Purchases $_{t}=\sum_{s \in S, v \in V_{t r}, w \in V_{g}, p \in P}\left(\mathrm{pb}_{s} \cdot\left(\mathrm{rm}_{p, w, t} \cdot \mathrm{PU}_{v, w, p, s, t}\right)\right) \quad \forall t \in T$

Constraint $62_{t}$

Inventory $_{t}=\sum_{s \in S, v \in \mathrm{V}}\left(\mathrm{pb}_{s} \cdot\left(\operatorname{Cinv}_{v, t} \cdot \mathrm{IL}_{v, s, t}\right)\right) \quad \forall t \in T$ 
Constraint $63_{t}$

Sales $_{t}=\sum_{s \in S, v \in V_{v}, p \in P}\left(\mathrm{pb}_{s} \cdot\left(\right.\right.$ SPrice $\left.\left._{p, v, t} \cdot \mathrm{SA}_{v, p, s, t}\right)\right) \quad \forall t \in T$

Constraint $64_{v, w, p, s, t}$

$\mathrm{PU}_{v, w, p, s, t}=\mathrm{Q}_{w, v, p, s, t}$

$\forall v \in V_{f}, w \in V_{g}, p \in P, t \in T, s \in \mathrm{N}_{t}$

Constraint $65_{p, v, s, t}$

$\mathrm{ID}_{p, v, s, t}=\mathrm{D}_{p, v, s, t}-\sum_{w \in \mathrm{F}_{w, v}} \mathrm{Q}_{w, v, p, s, t} \quad \forall p \in P, v \in V_{m}, t \in T, s \in \mathrm{N}_{t}$

Constraint $66_{t}$

$E S C=\frac{\sum_{t \in T}\left(1-\sum_{s} p b_{s}\left[\frac{\sum_{v \in V_{m}} \sum_{p \in P} I D_{p v s t}}{\sum_{v \in V_{m}} \sum_{p \in P} D_{p v s t}}\right]\right)}{|T|}$

Constraint $67_{t}$

Invest $_{t}=\sum_{i \in I, v \in V_{c}}\left(\mathrm{v}^{p}{ }_{i, v, t} \cdot \mathrm{CE}_{i, v, t}\right)+\sum_{i \in I, v \in V}\left(\mathrm{CPLinit}_{i, v} \cdot \mathrm{in}_{i, v}\right)+\sum_{v \in \mathrm{V}}\left(\mathrm{in}_{v}^{s} \cdot \mathrm{is}_{v}\right)+\sum_{v \in \mathrm{V}}\left(\mathrm{v}_{v, t}^{s} \cdot\right.$

$\left.\mathrm{CE}^{s}{ }_{v, t}\right)$

$\forall t \in T \wedge(t=1)$

Constraint $68_{t}$

Invest $_{t}=\sum_{i \in I, v \in V_{c}}\left(\mathrm{v}^{p}{ }_{i, v, t} \cdot \mathrm{CE}_{i, v, t}\right)+\sum_{v \in \mathrm{V}}\left(\mathrm{v}_{v, t}^{s} \cdot \mathrm{CE}_{v, t}^{s}\right) \quad \forall t \in T \wedge(t>1)$

Constraint $69_{t}$

$\mathrm{CFtot}_{t}=\sum_{s \in \mathrm{N}_{t}}\left(\mathrm{pb}_{s} \cdot \mathrm{CF}_{s, t}\right)$

Constraint $72_{v, t}$

$\sum_{w \in \mathrm{F}_{v, w}} \mathrm{Y}_{v, w, t}+\sum_{w \in \mathrm{R}_{v, w}}\left(\mathrm{YNC}_{v, w, t}+\mathrm{YEL}_{v, w, t}\right)+\sum_{w \in \mathrm{F}_{w, v}} \mathrm{Y}_{w, v, t}+\sum_{w \in \mathrm{R}_{w, v}}\left(\mathrm{YNC}_{w, v, t}+\right.$

$\left.\mathrm{YEL}_{w, v, t}\right)-99999 \cdot \mathrm{Xnodes}_{v, t} \leq 0$

$\forall v \in V, t \in T$

Constraint $73_{v, t}$

$\sum_{w \in \mathrm{F}_{v, w}} \mathrm{Y}_{v, w, t}+\sum_{w \in \mathrm{R}_{v, w}}\left(\mathrm{YNC}_{v, w, t}+\mathrm{YEL}_{v, w, t}\right)+\sum_{w \in \mathrm{F}_{w, v}} \mathrm{Y}_{w, v, t}+\sum_{w \in \mathrm{R}_{w, v}}\left(\mathrm{YNC}_{w, v, t}+\right.$

$\left.\mathrm{YEL}_{w, v, t}\right) \geq \operatorname{Xnodes}_{v, t}$ $\forall v \in V, t \in T$

Constraint 74

$\mathrm{NPV}$ total $=\sum_{s \in S}\left(\mathrm{pb}_{s} \cdot \mathrm{NPV}_{s}\right)$ 


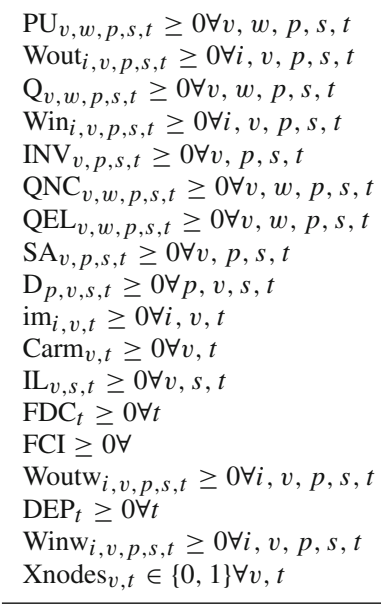

\section{References}

Adobor, H. (2019). Supply chain resilience: A multi-level framework. International Journal of Logistics Research and Applications, 22(6), 533-556. https://doi.org/10.1080/13675567.2018.1551483

Air (2020). Covid-19 is closing borders and attitudes as globalisation slows down. Retrieved 4-7-2020. https:// www.airport-technology.com/comment/covid-19-closing-borders-globalisation/.

Albertzeth, G., Nyoman Pujawan, I., Hilletofth, P., \& Tjahjono, B. (2020). Mitigating transportation disruptions in a supply chain: A cost-effective strategy. International Journal of Logistics Research and Applications, 23(2), 139-158. https://doi.org/10.1080/13675567.2019.1648640

Asian, S., \& Nie, X. (2014). Coordination in supply chains with uncertain demand and disruption risks: Existence, analysis, and insights. IEEE Transactions on Systems, Man, and Cybernetics: Systems, 44(9), 1139-1154.

Azad, N., Saharidis, G. K. D., Davoudpour, H., Malekly, H., \& Yektamaram, S. A. (2013). Strategies for protecting supply chain networks against facility and transportation disruptions: An improved Benders decomposition approach. Annals of Operations Research, 210(1), 125-163.

Azevedo, S. G., Carvalho, H., \& Cruz-Machado, V. (2016). LARG index: A benchmarking tool for improving the leanness, agility, resilience and greenness of the automotive supply chain. Benchmarking: An International Journal, 23(6), 1472-1499.

Baldwin, R, \& Tomiura, E. (2020). Thinking ahead about the trade impact of COVID-19. Economics in the Time of COVID-19 59.

Barroso, A. P., Cruz Machado, V, \& Machado, V. H. (2011). Supply chain resilience using the mapping approach. INTECH Open Access Publisher.

Bernardes, E. S., \& Hanna, M. D. (2009). A theoretical review of flexibility, agility and responsiveness in the operations management literature: Toward a conceptual definition of customer responsiveness. International Journal of Operations and Production Management, 29(1), 30-53.

Birkie, S. E. (2016). Operational resilience and lean: In search of synergies and trade-offs. Journal of Manufacturing Technology Management, 27(2), 185-207.

Brusset, X., \& Bertrand, J. L. (2018). Hedging weather risk and coordinating supply chains. Journal of Operations Management, 64(October), 41-52. https://doi.org/10.1016/j.jom.2018.10.002

Cardoso, S. R., Barbosa-Póvoa, A. P., Relvas, S., \& Novais, A. Q. (2015). Resilience metrics in the assessment of complex supply-chains performance operating under demand uncertainty. Omega, 56, 53-73.

Carvalho, H., Barroso, A. P., Machado, V. H., Azevedo, S., \& Cruz-Machado, V. (2012). Supply chain redesign for resilience using simulation. Computers \&amp; Industrial Engineering, 62(1), 329-341.

Carvalho, H., Duarte, S., \& Machado, V. C. (2011). Lean, agile, resilient and green: Divergencies and synergies. International Journal of Lean Six Sigma, 2(2), 151-179.

Chen, L., \& Bin, H. (2017). Is Reshoring better than offshoring? The effect of offshore supply dependence. Manufacturing and Service Operations Management, 19(2), 166-184. 
Cohen, M. A., \& Lee, H. L. (2020). Designing the right global supply chain network. Manufacturing and Service Operations Management, 22(1), 15-24.

Das, K. (2018). Integrating resilience in a supply chain planning model. International Journal of Quality and Reliability Management, 35(3), 570-595. https://doi.org/10.1108/IJQRM-08-2016-0136

Demirel, S., Kapuscinski, R., \& Man, Y. (2018). Strategic behavior of suppliers in the face of production disruptions. Management Science, 64(2), 533-551.

Dolgui, A., Ivanov, D., \& Sokolov, B. (2018). Ripple effect in the supply chain: An analysis and recent literature. International Journal of Production Research, 56(1-2), 414-430. https://doi.org/10.1080/ 00207543.2017 .1387680

Duhamel, C., Santos, A. C., Brasil, D., Châtelet, E., \& Birregah, B. (2016). Connecting a population dynamic model with a multi-period location-allocation problem for post-disaster relief operations. Annals of Operations Research, 247(2), 693-713.

Elleuch, H., Dafaoui, E., Elmhamedi, A., \& Chabchoub, H. (2016). Resilience and vulnerability in supply chain: Literature review. IFAC-Papers OnLine, 49(12), 1448-1453.

Elluru, S., Gupta, H., Kaur, H., \& Singh, S. P. (2019). Proactive and reactive models for disaster resilient supply chain. Annals of Operations Research, 283(1-2), 199-224. https://doi.org/10.1007/s10479-0172681-2

Fahimnia, B., \& Jabbarzadeh, A. (2016). Marrying supply chain sustainability and resilience: A match made in heaven. Transportation Research Part E: Logistics and Transportation Review, 91, 306-324.

Farahani, R. Z., Hekmatfar, M., Arabani, A. B., \& Nikbakhsh, E. (2013). Hub location problems: A review of models, classification, solution techniques, and applications. Computers \&amp; Industrial Engineering, 64(4), 1096-1109.

Farahani, R. Z., Hekmatfar, M., Fahimnia, B., \& Narges, K. (2014). Hierarchical facility location problem: Models, classifications, techniques, and applications. Computers and Industrial Engineering, 68(1), 104117.

Fitch Solutions. (2020). "Covid-19 Pandemic Exposed Over-Reliance On China For Pharmaceutical Raw Materials. Retrieved 3-7-2020. https://www.fitchsolutions.com/corporates/healthcare-pharma/covid19-pandemic-exposed-over-reliance-china-pharmaceutical-raw-materials-26-05-2020.

Gaonkar, R. S., \& Viswanadham, N. (2007). Analytical framework for the management of risk in supply chains. IEEE Transactions on Automation Science and Engineering, 4(2), 265-273.

Gereffi, G. (2020). What does the COVID-19 pandemic teach us about global value chains? The case of medical supplies. Journal of International Business Policy. https://doi.org/10.1057/s42214-020-00062$\mathrm{W}$

Goffnett, S. P., \& Williams, Z. (2019). The path between supply chain efficacy and performance: Testing a secure route. International Journal of Logistics Research and Applications, 22(1), 98-117. https://doi. org/10.1080/13675567.2018.1475555

Golan, M. S., Jernegan, L. H., \& Linkov, I. (2020). Trends and applications of resilience analytics in supply chain modeling: Systematic literature review in the context of the COVID-19 pandemic. Environment Systems and Decisions, 40(2), 222-243. https://doi.org/10.1007/s10669-020-09777-w

Ha, C., Jun, H.-B., \& Ok, C. (2018). A mathematical definition and basic structures for supply chain reliability: A procurement capability perspective. Computers \&amp; Industrial Engineering, 120, 334.

Han, J., \& Shin, K. (2016). Evaluation mechanism for structural robustness of supply chain considering disruption propagation. International Journal of Production Research, 54(1), 135-151.

Henry, D., \& Ramirez-Marquez, J. E. (2012). Generic metrics and quantitative approaches for system resilience as a function of time. Reliability Engineering and System Safety, 99, 114-122. https://doi.org/10.1016/ j.ress.2011.09.002

Hobbs, J. E. (2020). Food supply chains during the COVID-19 pandemic. Canadian Journal of Agricultural Economics, 68, 171.

Hohenstein, N.-O., Feisel, E., Hartmann, E., \& Giunipero, L. (2015). Research on the phenomenon of supply chain resilience: A systematic review and paths for further investigation. International Journal of Physical Distribution \&amp; Logistics Management, 45(1/2), 90-117.

Hosseini, S., \& Ivanov, D. (2019). A new resilience measure for supply networks with the ripple effect considerations: A Bayesian network approach. Annals of Operations Research. https://doi.org/10.1007/ s10479-019-03350-8

Hosseini, S., Ivanov, D., \& Dolgui, A. (2019). Review of quantitative methods for supply chain resilience analysis. Transportation Research Part E: Logistics and Transportation Review, 125, 285-307.

Howard, M., Hopkinson, P., \& Miemczyk, J. (2018). The regenerative supply chain: A framework for developing circular economy indicators. International Journal of Production Research. https://doi.org/10.1080/ 00207543.2018 .1524166 
Ivanov, D., \& Dolgui, A. (2018). Low-Certainty-Need (LCN) supply chains: A new perspective in managing disruption risks and resilience. International Journal of Production Research, 57(15-16), 5119-5136. https://doi.org/10.1080/00207543.2018.1521025

Ivanov, D., Dolgui, A., Sokolov, B., \& Ivanova, M. (2017). Literature review on disruption recovery in the supply chain*. International Journal of Production Research, 55(20), 6158-6174.

Jahani, H., Abbasi, B., Hosseinifard, Z., Fadaki, M., \& Minas, J. P. (2020). Disruption risk management in service-level agreements. International Journal of Production Research, 1, 1-19.

Jewkes, Stephen, \& Amante, Angelo. (2020). Italy to reopen factories in staged end to coronavirus lockdown. Accessed 2-7-2020. https://www.reuters.com/article/us-health-coronavirus-italy-conte/italy-toreopen-factories-in-staged-end-to-coronavirus-lockdown-idUSKCN22807C.

Kamalahmadi, M., \& Parast, M. M. (2016). A review of the literature on the principles of enterprise and supply chain resilience: Major findings and directions for future research. International Journal of Production Economics, 171, 116-133.

Kaur, H., \& Singh, S. P. (2019). Sustainable procurement and logistics for disaster resilient supply chain. Annals of Operations Research, 283(1-2), 309-354.

Kim, Y., Chen, Y.-S., \& Linderman, K. (2015). Supply network disruption and resilience: A network structural perspective. Journal of Operations Management, 33, 43-59.

Kirwan, J., Maye, D., \& Brunori, G. (2017). Acknowledging complexity in food supply chains when assessing their performance and sustainability. Journal of Rural Studies, 52, 21-32.

König, A., \& Spinler, S. (2016). The effect of logistics outsourcing on the supply chain vulnerability of shippers: Development of a conceptual risk management framework. The International Journal of Logistics Management, 27(1), 122-141.

Li, G., Li, L., Zhou, Y., \& Guan, X. (2017). Capacity restoration in a decentralized assembly system with supply disruption risks. International Transactions in Operational Research, 24(4), 763-782.

Li, G., Liu, M., Bian, Y., \& Sethi, S. P. (2020). Guarding against disruption risk by contracting under information asymmetry. Decision Sciences, 00, 1-39.

Lotfi, M., \& Saghiri, S. (2017). Disentangling resilience, agility and leanness. Journal of Manufacturing Technology Management.

Lussenhop, J. (2020). Coronavirus at Smithfield pork plant: The untold story of America's biggest outbreak. Accessed 2-7-2020. https://www.bbc.com/news/world-us-canada-52311877.

Mari, S. I., Lee, Y. H., \& Memon, M. S. (2014). Sustainable and resilient supply chain network design under disruption risks. DSustainability, 6(10), 6666-6686.

Mohammed, A., Harris, I., Soroka, A., Naim, M., Ramjaun, T., \& Yazdani, Morteza. (2021). Gresilient supplier assessment and order allocation planning. Annals of Operations Research, 296(1), 335-362.

Mota, B., Gomes, M. I., Carvalho, A., \& Barbosa-Póvoa, A. P. (2015). Towards supply chain sustainability: Economic, environmental and social design and planning. Journal of Cleaner Production, 105, 14-27.

Mota, B., Gomes, M. I., Carvalho, A., \& Barbosa-Póvoa, A. P. (2017). Sustainable supply chains: An integrated modeling approach under uncertainty. Omega., 77, 32.

Mullin, R. (2020). COVID-19 is reshaping the pharmaceutical supply chain. Retrieved 3-7-2020. https://cen. acs.org/business/outsourcing/COVID-19-reshaping-pharmaceutical-supply/98/i16.

Munoz, A., \& Dunbar, M. (2015). On the quantification of operational supply chain resilience. International Journal of Production Research, 53(22), 6736-6751.

Mwangi, G. M., Despoudi, S., Espindola, O. R., Spanaki, K., \& Papadopoulos, T. (2021). A planetary boundaries perspective on the sustainability: Resilience relationship in the Kenyan tea supply chain. Annals of Operations Research, 18, 1-35.

Ning, C., \& You, F. (2018). Adaptive robust optimization with minimax regret criterion: Multiobjective optimization framework and computational algorithm for planning and scheduling under uncertainty. Computers \&amp; Chemical Engineering, 108, 425-447.

Oliver, R. K., \& Webber, M. D. (1982). Supply-chain management: Logistics catches up with strategy. Outlook, 5(1), 42-47.

Özçelik, G., Yılmaz, Ö. F., \& Yeni, F. B. (2020). Robust optimisation for ripple effect on reverse supply chain: An industrial case study. International Journal of Production Research. https://doi.org/10.1080/ 00207543.2020 .1740348

Paul, S. K., \& Chowdhury, P. (2020). A production recovery plan in manufacturing supply chains for a high-demand item during COVID-19. International Journal of Physical Distribution and Logistics Management.

Ribeiro, J., \& Barbosa-Póvoa, A. (2019). Supply Chain Resilience: An optimisation model to identify the relative importance of sc disturbances. In Operational Research. https://doi.org/10.1007/978-3-03010731-4_13 
Ponis, S. T., \& Koronis, E. (2012). Supply chain resilience: Definition of concept and its formative elements. Journal of Applied Business Research, 28(5), 921.

Ponomarov, S. (2012). Antecedents and consequences of supply chain resilience: A dynamic capabilities perspective.

Ponomarov, S. Y., \& Holcomb, M. C. (2009). Understanding the concept of supply chain resilience. The International Journal of Logistics Management, 20(1), 124-143.

Rajesh, R., \& Ravi, V. (2015). Modeling enablers of supply chain risk mitigation in electronic supply chains: A Grey-DEMATEL approach. Computers and Industrial Engineering, 87, 126-139. https://doi.org/10. 1016/j.cie.2015.04.028

Ralston, P., \& Blackhurst, J. (2020). Industry 4.0 and resilience in the supply chain: A driver of capability enhancement or capability loss. International Journal of Production Research, 0(0), 1-14.

Ribeiro, João Pires, \& Barbosa-Póvoa, Ana. (2018a). Modelling and Analysing Supply Chain Resilience Flow Complexity. In 28th European Symposium on Computer Aided Process Engineering, edited by Anton Friedl, Jiř́ J. Klemeš, Stefan Radl, Petar S. Varbanov, and Thomas Wallek, Vol. 43 of Computer Aided Chemical Engineering, 815 - 820. Elsevier. http://www.sciencedirect.com/science/article/ pii/B9780444642356501431.

Ribeiro, J. P., \& Barbosa-Póvoa, A. (2018). Supply Chain Resilience: Definitions and quantitative modelling approaches: A literature review. Computers \&amp; Industrial Engineering, 115, 109-122.

Rice, J. B., \& Caniato, F. (2003). Building a secure and resilient supply network. Supply Chain Management Review, 7(5), 22-30.

RTE. (2020). Covid-19: Italy shuts factories after almost 800 deaths in one day. Accessed 2-7-2020. https:// www.rte.ie/news/2020/0321/1124574-italy-covid-19/.

Sabahi, S., \& Parast, M. M. (2020). Firm innovation and supply chain resilience: A dynamic capability perspective. International Journal of Logistics Research and Applications, 23(3), 254-269. https://doi. org/10.1080/13675567.2019.1683522

Sáenz, M. J., Revilla, E., \& Acero, B. (2018). Aligning supply chain design for boosting resilience. https:// www.sciencedirect.com/science/article/pii/S0007681318300090?via\%3Dihub.

Sahebjamnia, N., Torabi, S. A., \& Mansouri, S. A. (2015). Integrated business continuity and disaster recovery planning: Towards organizational resilience. European Journal of Operational Research, 242(1), 261273.

Savaskan, R. C., Bhattacharya, S., \& Van Wassenhove, L. N. (2004). Closed-loop supply chain models with product remanufacturing. Management Science, 50(2), 239-252.

Schmitt, A. J., \& Singh, M. (2012). A quantitative analysis of disruption risk in a multi-echelon supply chain. International Journal of Production Economics, 139(1), 22-32.

Sharma, N., Sahay, B. S., Shankar, R., \& Sarma, P. R. S. (2017). Supply chain agility: Review, classification and synthesis. International Journal of Logistics Research and Applications, 20(6), 532-559. https://doi. org/10.1080/13675567.2017.1335296

Shekarian, M., \& Parast, M. M. (2020). An Integrative approach to supply chain disruption risk and resilience management: A literature review. International Journal of Logistics Research and Applications. https:// doi.org/10.1080/13675567.2020.1763935

Singh, N. P. (2020). Managing environmental uncertainty for improved firm financial performance: The moderating role of supply chain risk management practices on managerial decision making. International Journal of Logistics Research and Applications, 23(3), 270-290. https://doi.org/10.1080/13675567.2019. 1684462

Snyder, L. V., Scaparra, M. P., Daskin, M. S., \& Church, R. L. (2006). Planning for disruptions in supply chain networks. INFORMS.

Sokolov, B., Ivanov, D., Dolgui, A., \& Pavlov, A. (2016). Structural quantification of the ripple effect in the supply chain. International Journal of Production Research, 54(1), 152-169.

Song, G., \& Sun, L. (2017). Evaluation of factors affecting strategic supply chain network design. International Journal of Logistics Research and Applications, 20(5), 405-425. https://doi.org/10.1080/13675567. 2016.1267125

Soni, U., Vipul Jain, V. B., \& Sameer Kumar, S. C. (2014). Measuring supply chain resilience using a deterministic modeling approach. Computers and Industrial Engineering, 74(1), 11-25. https://doi.org/10. 1016/j.cie.2014.04.019

Srinivasan, R. S., \& Tew, J. D. (2017). Supply chain immune system: Concept, framework, and applications. International Journal of Logistics Research and Applications, 20(6), 515-531. https://doi.org/10.1080/ 13675567.2017.1324834

Stone, J., \& Rahimifard, S. (2018). Resilience in agri-food supply chains: A critical analysis of the literature and synthesis of a novel framework. Supply Chain Management: An International Journal. https://doi. org/10.1108/SCM-06-2017-0201 
Takahashi, Y. (2011). Japan's Car Production Surpasses Pre-Quake Level. sep. https://blogs.wsj.com/driversseat/2011/09/30/japans-car-production-surpasses-pre-quake-level/?mod=searchresults \&page=7\& pos $=14$.

Tang, C. S. (2006). Perspectives in supply chain risk management. International Journal of Production Economics, 103(2), 451-488.

Tao, Y., Lai, X., \& Zhou, S. (2020). Information sharing in a transparent supply chain with transportation disruptions and supplier competition. Annals of Operations Research. https://doi.org/10.1007/s10479020-03724-3

Vigdor, J. (2008). The economic aftermath of hurricane katrina. Journal of Economic Perspectives, 22(4), 135-54.

Wachtendorf, T., Brown, B., \& Holguin-Veras, J. (2013). Catastrophe characteristics and their impact on critical supply chains: Problematizing materiel convergence and management following hurricane katrina. Journal of Homeland Security and Emergency Management, 10(2), 497-520.

Wakasugi, R., \& Tanaka, A. (2015). Recovery of the supply chain after the 2011 mega-quake in Japan. Millennial Asia, 6(1), 1-18.

Wang, D., \& Ip, W. H. (2009). Evaluation and analysis of logistic network resilience with application to aircraft servicing. IEEE Systems Journal, 3(2), 166-173.

Wang, J., Muddada, R. R., Wang, H., Ding, J., Lin, Y., Liu, C., \& Zhang, W. (2016). Toward a resilient holistic supply chain network system: Concept, review and future direction. IEEE Systems Journal, 10(2), 410421.

Wimmer, T. (2020). The Covid-19 crisis and its impact on supply chain management in Germany and Europe. Retrieved 4-7-2020. https://www.eurologport.eu/the-covid-19-crisis-and-its-impact-on-supply-chainmanagement-in-germany-and-europe/.

Publisher's Note Springer Nature remains neutral with regard to jurisdictional claims in published maps and institutional affiliations. 